\title{
Time Local Well-posedness for the Benjamin-Ono Equation with Large Initial Data
}

\author{
By \\ Naoyasu KITA* and Jun-ichi SEgATA**
}

\begin{abstract}
This paper studies the time local well-posedness of the solution to the BenjaminOno equation. Our aim is to remove smallness condition on the initial data which was imposed in Kenig-Ponce-Vega's work [13].
\end{abstract}

\section{$\S 1 . \quad$ Introduction}

We consider the initial value problem for the Benjamin-Ono equation:

$$
\left\{\begin{array}{lr}
\partial_{t} u+\mathcal{H}_{x} \partial_{x}^{2} u+u \partial_{x} u=0, & x, t \in \mathbf{R}, \\
u(x, 0)=u_{0}(x), & x \in \mathbf{R},
\end{array}\right.
$$

where $\mathcal{H}_{x}$ denotes the Hilbert transform, i.e., $\mathcal{H}_{x}=\mathcal{F}^{-1}(-i \xi /|\xi|) \mathcal{F}$. The equation (1.1) arises in the study of long internal gravity waves in deep stratified fluid. For the physical background, see Benjamin [3] and Ono [18].

We present the time local well-posedness of (1.1). Namely, we prove the existence, uniqueness of the solution and the continuous dependence on the initial data. There are several known results about this problem. One of their concern is to overcome the regularity loss arising from the nonlinearity. Because of this difficulty, the contraction mapping principle via the associated integral

Communicated by H. Okamoto. Received March 22, 2004. Revised October 19, 2004.

2000 Mathematics Subject Classification(s): 35Q53.

Key words: Benjamin-Ono equation, time local well-posedness, smoothing effect.

* Faculty of Education and Culture, Miyazaki University, Nishi 1-1, Gakuen kiharudai, Miyazaki 889-2192, Japan.

e-mail: nkita@cc.miyazaki-u.ac.jp

** Graduate School of Mathematics, Kyushu University and Mathematical Institute, Tohoku University, Aoba, Sendai, 980-8578, Japan.

e-mail: segata@math.tohoku.ac.jp 
equation does not work as long as we consider the estimates only in the Sobolev space $H_{x}^{s, 0}$, where $H_{x}^{s, \alpha}$ is defined by

$$
H_{x}^{s, \alpha}=\left\{f \in \mathcal{S}^{\prime}(\mathbf{R}) ;\|f\|_{H_{x}^{s, \alpha}}<\infty\right\}
$$

with $\|f\|_{H_{x}^{s, \alpha}}=\left\|\langle x\rangle^{\alpha}\left\langle D_{x}\right\rangle^{s} f\right\|_{L_{x}^{2}},\langle x\rangle^{\alpha}=\left(1+x^{2}\right)^{\alpha / 2}$ and $\left\langle D_{x}\right\rangle^{s}=\mathcal{F}^{-1}\langle\xi\rangle^{s} \mathcal{F}$. Indeed, Molinet-Saut-Tzvetkov [17] negatively proved the solvability of the integral equation in $H_{x}^{s, 0}$ for any $s \in \mathbf{R}$.

Saut [21] proved the global well-posedness for (1.1) in $H_{x}^{3}$. AbdelouhabBona-Felland-Saut [1] and Iorio [8] proved the time global existence and uniqueness of the solution in $H_{x}^{s, 0}$ with $s>3 / 2$. Their proofs are based on the energy method in which the estimate of $\left\|\partial_{x} u\right\|_{L_{T}^{\infty}\left(L_{x}^{\infty}\right)}$ gives the regularity constraint of the initial data. Ponce [19] obtained the global unique solution in $H_{x}^{3 / 2,0}$ by the combination of energy method and dispersive structure of linear part in (1.1). More recently, Koch-Tzvetkov [14] have studied the local well-posedness with $s>5 / 4$ due to the cut off technique of $\mathcal{F} u(\xi)$. Furthermore, Kenig-Koenig [10] proved the local well-posedness with $s>9 / 8$. We remark here that it is possible to minimize the regularity of $u_{0}$ by inducing another kind of function space. In fact, Kenig-Ponce-Vega [13] construct a time local solution via the integral equation by applying the smoothing property like

$$
\left\|D_{x} \int_{0}^{t} V\left(t-t^{\prime}\right) F\left(t^{\prime}\right) d t^{\prime}\right\|_{L_{x}^{\infty}\left(L_{T}^{2}\right)} \leq C\|F\|_{L_{x}^{1}\left(L_{T}^{2}\right)},
$$

where $\|u\|_{L_{x}^{p}\left(L_{T}^{r}\right)}=\left\|\left(\|u\|_{L^{r}[0, T]}\right)\right\|_{L_{x}^{p}(\mathbf{R})}, \quad D_{x}=\mathcal{F}^{-1}|\xi| \mathcal{F}$ and $V(t)=$ $\exp \left(-t \mathcal{H}_{x} \partial_{x}^{2}\right)$. They obtained the time local well-posedness in $H_{x}^{s, 0}(s>1)$ for the cubic nonlinearity (Their argument is also applicable to the quadratic case if $u_{0}$ satisfies $u_{0} \in H_{x}^{s, 0}(s>1)$ and the additional weight condition). In their result, however, the smallness of the initial data is required. This is because the inclusion $L_{x}^{1}\left(L_{T}^{\infty}\right) \cdot L_{x}^{\infty}\left(L_{T}^{2}\right) \subset L_{x}^{1}\left(L_{T}^{2}\right)$ yields $\|u\|_{L_{x}^{1}\left(L_{T}^{\infty}\right)}$ in the nonlinearity and we can not expect that $\|u\|_{L_{x}^{1}\left(L_{T}^{\infty}\right)} \rightarrow 0$ even when $T \rightarrow 0$.

Our concern in this paper is to remove this smallness condition of $u_{0}$. Before presenting the rough sketch of our idea, we introduce the function space $Y_{T}$ in which the solution is constructed:

$$
Y_{T}=\left\{u:[0, T] \times \mathbf{R} \rightarrow \mathbf{R} ;\|u\|_{Y_{T}}<\infty\right\},
$$

where $\|u\|_{Y_{T}}=\|u\|_{L_{T}^{\infty}\left(H_{x}^{s, 0} \cap H_{x}^{s_{1}, \alpha_{1}}\right)}+\left\|\langle x\rangle^{-\rho}\left\langle D_{x}\right\rangle^{s+1 / 2} u\right\|_{L_{x}^{1 / \varepsilon}\left(L_{T}^{2}\right)}+$ $\left\|\left\langle D_{x}\right\rangle^{\mu}\langle x\rangle^{\alpha_{1}} u\right\|_{L_{x}^{2}\left(L_{T}^{\infty}\right)}$ with $\rho, \mu>0$ sufficiently small and $0<\varepsilon<\rho$. We first consider the modified equation such that

$$
\left\{\begin{array}{l}
\partial_{t} u_{\nu}+\mathcal{H}_{x} \partial_{x}^{2} u_{\nu}+u_{\nu} \partial_{x} \eta_{\nu} * u_{\nu}=0 \\
u_{\nu}(0, x)=u_{0}(x)
\end{array}\right.
$$


where $\eta_{\nu}(x)=\nu^{-1} \eta(x / \nu)$ with $\eta \in C_{0}^{\infty}, \int \eta(x) d x=1$ and $\nu \in(0,1]$. Then, the existence of $u_{\nu}$ in $Y_{T}$ easily follows and it is continuated as long as $\left\|u_{\nu}(t)\right\|_{H_{x}^{s, 0} \cap H_{x}^{s_{1}, \alpha_{1}}}<\infty$. Note that $\left\|u_{\nu}\right\|_{Y_{T}}$ is continuous with respect to $T$. To seek for the a priori estimate of $\left\|u_{\nu}\right\|_{Y_{T}}$, we deform (1.2). Let $\varphi \in C_{0}^{\infty}(\mathbf{R})$ and write $u_{\nu} \partial_{x} \eta_{\nu} * u_{\nu}=\varphi \partial_{x} \eta_{\nu} * u_{\nu}+\left(u_{\nu}-\varphi\right) \partial_{x} \eta_{\nu} * u_{\nu}$. Note here that, if $\varphi$ is close to $u_{0}$, one can make $u_{\nu}-\varphi$ sufficiently small when $t \rightarrow 0$. To control the heavy term $\varphi \partial_{x} \eta_{\nu} * u_{\nu}$, we employ the gauge transform (see section 2) so that this quantity is, roughly speaking, absorbed in the linear operator. Then, our desired a priori estimate follows via the integral equation. As for the convergence of nonlinearity $u_{\nu} \partial_{x} \eta_{\nu} * u_{\nu} \rightarrow u \partial_{x} u$, we also consider the estimate of $u_{\nu}-u_{\nu^{\prime}}$ in $Y_{T}^{\prime}$ which is slightly weaker than $Y_{T}$ (see Proposition 6.1). Let us now state our main theorem.

Theorem 1.1. (i) Let $u_{0} \in H_{x}^{s, 0} \cap H_{x}^{s_{1}, \alpha_{1}} \equiv X^{s}$ with $s_{1}+\alpha_{1}<s, 1 / 2<$ $s_{1}$ and $1 / 2<\alpha_{1}<1$. Then, for some $T=T\left(u_{0}\right)>0$, there exists a unique solution to (1.1) such that $u \in C\left([0, T] ; X^{s}\right) \cap Y_{T}$.

(ii) Let $u^{\prime}(t)$ be the solution to (1.1) with the initial data $u_{0}^{\prime}$ satisfying $\| u_{0}^{\prime}-$ $u_{0} \|_{X^{s}}<\delta$. If $\delta>0$ is sufficiently small, then there exist some $T^{\prime} \in(0, T)$ and $C>0$ such that

$$
\begin{aligned}
& \left\|u^{\prime}-u\right\|_{L_{T^{\prime}}^{\infty}\left(X^{s}\right)} \leq C\left\|u_{0}^{\prime}-u_{0}\right\|_{X^{s}}, \\
& \left\|\langle x\rangle^{-\rho}\left\langle D_{x}\right\rangle^{s+1 / 2}\left(u^{\prime}-u\right)\right\|_{L_{x}^{1 / \varepsilon}\left(L_{T^{\prime}}^{2}\right)} \leq C\left\|u_{0}^{\prime}-u_{0}\right\|_{X^{s}} .
\end{aligned}
$$

In Theorem 1.1, the conditions on the initial data are determined by the estimate of maximal function, where, we call $\|f(\cdot, x)\|_{L_{T}^{\infty}}$ the maximal function of $f(t, x)$. Concretely speaking, the quantity $\|u\|_{L_{x}^{1}\left(L_{T}^{\infty}\right)}$ is bounded by $C\left(\left\|u_{0}\right\|_{H_{x}^{s, 0}}+\left\|u_{0}\right\|_{H_{x}^{s_{1}, \alpha_{1}}}\right)$ (see Lemma 4.2).

Remark 1. Only for the existence, one can further minimize the regularity of the initial data. Abdelouhab-Bona-Fell-Saut [1], Ginibre-Velo [7], Saut [21] and Tom [23] proved the global existence of weak solutions in $L_{x}^{2}, H_{x}^{1 / 2,0}$ and $H_{x}^{1,0}$, respectively. Recently, Tao [22] has studied the global well-posedness in $H_{x}^{1,0}$ but the $L^{2}$-stability of the data-to-solution map holds while the initial data belongs to $H_{x}^{1,0}$, i.e., $\left\|u^{\prime}(t)-u(t)\right\|_{L^{2}} \leq C\left\|u_{0}^{\prime}-u_{0}\right\|_{L^{2}}$. More recently, Kato [9] has proved the well-posedness by supposing that $u_{0} \in H_{x}^{s}$ with $s>1 / 2$ and roughly speaking, $u_{0}$ satisfies the zero average condition $\int u_{0}(x) d x=0$.

We also remark that Koch-Tzvetkov [17] and Biagioni-Linares [5] negatively proved the strong stability like

$$
\left\|u^{\prime}(t)-u(t)\right\|_{H_{x}^{s, 0}} \leq C\left\|u_{0}^{\prime}-u_{0}\right\|_{H_{x}^{s, 0}} \quad \text { for } s>0
$$


if there is no weight condition on $u_{0}$ and $u_{0}^{\prime}$. Though our result requires slightly large regularity in comparison with Tao's work, it suggests that the additional weight condition yields the strong stability of the data-to-solution map in the sense that its target space coincides with that of initial data.

Remark 2. The upper bound of $\alpha_{1}$ is required in the proof of weighted norm estimates (see section 4) and especially, in the estimate of $\left[\langle x\rangle^{\alpha_{1}}, \mathcal{H}_{x} \partial_{x}^{2}\right]$. It is possible to relax this weight condition. However, for the simplicity of our argument, we do not handle this kind of generalization in this paper. Let us also remark that the persistence of the solution fails if $\alpha_{1} \geq 3$ (see Iorio [8]).

We close this section by introducing several notations and reviewing typical facts on the pseudo-differential operators. The Fourier transform $(2 \pi)^{-1 / 2}$ $\int e^{-i x \xi} f(x) d x$ is denoted by $\mathcal{F} f$ or $\hat{f} . \mathcal{B}(X ; Y)$ stands for the class of bounded operators from $X$ to $Y$. For simplicity, we often write $\mathcal{B}(X ; X)=\mathcal{B}(X)$. The norm of the summation space $X+Y$ is given by $\|f\|_{X+Y}=\inf \left\{\|g\|_{X}+\|h\|_{Y} ; g+\right.$ $h=f\}$. We call $a(x, \xi) \in C^{\infty}(\mathbf{R} \times \mathbf{R})$ belongs to the symbol class $S^{m}$ if $\sup _{x, \xi}\langle\xi\rangle^{-m+j}\left|\partial_{\xi}^{j} \partial_{x}^{k} a(x, \xi)\right|<\infty$. For this symbol, the pseudo-differential operator $a\left(x, i^{-1} \partial_{x}\right)$ is defined by

$$
a\left(x, i^{-1} \partial_{x}\right) f=(2 \pi)^{-1 / 2} \int e^{i x \xi} a(x, \xi) \hat{f}(\xi) d \xi .
$$

Let $\sigma\left(a\left(x, i^{-1} \partial_{x}\right)\right)$ be the symbol of $a\left(x, i^{-1} \partial_{x}\right)$. It is well-known (cf. Kumanogo [16], Stein [20]) that, if $a(x, \xi) \in S^{\ell}$ and $b(x, \xi) \in S^{m}$, then we have

$$
\sigma\left(a\left(x, i^{-1} \partial_{x}\right) b\left(x, i^{-1} \partial_{x}\right)\right) \in S^{\ell+m} \text { and } \sigma\left(\left[a\left(x, i^{-1} \partial_{x}\right), b\left(x, i^{-1} \partial_{x}\right)\right]\right) \in S^{\ell+m-1} \text {, }
$$

where $[A, B]=A B-B A$. These properties follow from the symbolic expansion formula like

$$
\begin{aligned}
& \sigma\left(a\left(x, i^{-1} \partial_{x}\right) b\left(x, i^{-1} \partial_{x}\right)\right) \\
& =\sum_{j=0}^{N-1} \frac{1}{2 \pi j ! i^{j}} \partial_{\xi}^{j} a(x, \xi) \partial_{x}^{j} b(x, \xi) \\
& \quad+\frac{1}{2 \pi(N-1) ! i^{N}} \text { Os- } \iint e^{-i(x-y)(\xi-\zeta)} \partial_{\zeta} a(x, \zeta) \\
& \quad \times\left(\int_{0}^{1}(1-\theta)^{N-1} \partial_{x}^{N} b(\theta y+(1-\theta) x, \xi d \theta) d y d \zeta,\right.
\end{aligned}
$$

where Os- $\iint$ stands for the oscillatory integral with respect to $y$ and $\zeta$. The expansion formula (1.3) is also applicable even in the case $a(x, \xi)=\langle\xi\rangle^{\sigma}$ 
and $b(x, \xi)=\langle x\rangle^{\alpha}$, which gives the equivalence of $\left\|\langle x\rangle^{\alpha}\left\langle D_{x}\right\rangle^{\sigma} f\right\|_{L_{x}^{p}}$ (resp. $\left.\left\|\langle x\rangle^{\alpha}\left\langle D_{x}\right\rangle^{\sigma} f\right\|_{L_{x}^{p}\left(L_{T}^{r}\right)}\right)$ and $\left\|\left\langle D_{x}\right\rangle^{\sigma}\langle x\rangle^{\alpha} f\right\|_{L_{x}^{p}}$ (resp. $\left.\left\|\langle x\rangle^{\alpha}\left\langle D_{x}\right\rangle^{\sigma} f\right\|_{L_{x}^{p}\left(L_{T}^{r}\right)}\right)$. For the symbol $a(x, \xi) \in S^{m},|a|_{N}^{(m)}$ denotes the semi-norm defined by

$$
|a|_{N}^{(m)}=\max _{j+k \leq N} \sup _{x, \xi}\langle\xi\rangle^{-m+j}\left|\partial_{\xi}^{j} \partial_{x}^{k} a(x, \xi)\right| .
$$

We note that, for $a(x, \xi) \in S^{m}, b(x, \xi) \in S^{m^{\prime}}$ and arbitrary $N>0$, there exist some $N^{\prime}>0$ and $C>0$ such that

$$
\left|\sigma\left(a\left(x, i^{-1} \partial_{x}\right) b\left(x, i^{-1} \partial_{x}\right)\right)\right|_{N}^{\left(m+m^{\prime}\right)} \leq C|a|_{N^{\prime}}^{(m)}|b|_{N^{\prime}}^{\left(m^{\prime}\right)} .
$$

Furthermore, for $a(x, \xi) \in S^{m}, a\left(x, i^{-1} \partial_{x}\right) \in \mathcal{B}\left(L_{x}^{2}\right)$ if $m \leq 0$ and $a\left(x, i^{-1} \partial_{x}\right) \in$ $\mathcal{B}\left(L_{x}^{p}\right)(p \in[1, \infty])$ if $m<0$. In these estimates, we see that the operator norms $\left\|a\left(x, i^{-1} \partial_{x}\right)\right\|_{\mathcal{B}\left(L_{x}^{p}\right)}$ and $\left\|a\left(x, i^{-1} \partial_{x}\right)\right\|_{\mathcal{B}\left(L_{x}^{p}\left(L_{T}^{r}\right)\right)}$ are estimated by $|a|_{N}^{(m)}$ for some $N>0$. We also denote $\int_{0}^{t} V(t-\tau) F(\tau) d \tau$ by $G(t) F$.

\section{§2. Gauge Transform}

In this section, we transform (1.2) appropriately for the a priori estimate. We write

$$
\begin{aligned}
\partial_{t} u_{\nu}+\mathcal{H}_{x} \partial_{x}^{2} u_{\nu} & +\varphi \eta_{\nu} * \partial_{x} u_{\nu} \\
& +\left(u_{\nu}-\varphi\right) \eta_{\nu} * \partial_{x} u_{\nu}=0,
\end{aligned}
$$

with $\varphi \in C_{0}^{\infty}(\mathbf{R})$ to be chosen closely to $u_{0}$ in $H_{x}^{s, 0} \cap H_{x}^{s_{1}, \alpha_{1}}$. We next define the gauge transformation of pseudo-differential operator with the symbol:

$$
K_{\nu}(x, \xi)=\exp \left(\sqrt{\frac{\pi}{2}} \frac{i \xi}{|\xi|}(1-\psi(\xi)) \hat{\eta}(\nu \xi) \int_{-\infty}^{x} \varphi(y) d y\right)
$$

where $\psi \in C_{0}^{\infty}(\mathbf{R})$ satisfies

$$
\psi(\xi)= \begin{cases}1, & \text { if }|\xi|<1 \\ 0, & \text { if }|\xi|>2\end{cases}
$$

Note that $K_{\nu}(x, \xi) \in S^{0}$ uniformly in $\nu \in(0,1]$. Applying $K_{\nu} \equiv K_{\nu}\left(x, i^{-1} \partial_{x}\right)$ to (2.1) and letting $v_{\nu}=K_{\nu} u_{\nu}$, we have

$$
\partial_{t} v_{\nu}+\mathcal{H}_{x} \partial_{x}^{2} v_{\nu}+K_{\nu}\left(u_{\nu}-\varphi\right) \eta_{\nu} * \partial_{x} u_{\nu}+R_{\nu}\left(\varphi, u_{\nu}\right)=0
$$

where $R_{\nu}(\varphi, u)=\left(K_{\nu} \varphi \eta_{\nu} * \partial_{x}+\left[K_{\nu}, \mathcal{H}_{x} \partial_{x}^{2}\right]\right) u$. Note that the symbol of $K_{\nu} \varphi \eta_{\nu} *$ $\partial_{x}+\left[K_{\nu},\left(1-\psi\left(i^{-1} \partial_{x}\right)\right) \mathcal{H}_{x} \partial_{x}^{2}\right]$ belongs to $S^{0}$ uniformly in $\nu \in(0,1]$ since the 
top symbol of the commutator is $-\partial_{x} K_{\nu}(x, \xi) \partial_{\xi}((1-\psi) \xi|\xi|)$. The desired a priori estimate of $u_{\nu}$ will be obtained in terms of $v_{\nu}$ by transforming (2.3) into the integral equation. Henceforth, we are led to the preliminaries about several estimates of $V(t)$ etc. (These are given in the next section.)

\section{§3. Preliminary}

We introduce several linear estimates. The first lemma gives the smoothing effects due to Kenig-Ponce-Vega [13] which overcome a loss of regularity in the nonlinearity.

Lemma 3.1. Let $p \in[2, \infty]$. Then we have

$$
\begin{aligned}
\left\|D_{x}^{1 / 2-1 / p} V(t) \phi\right\|_{L_{x}^{p}\left(L_{T}^{2}\right)} & \leq C T^{1 / p}\|\phi\|_{L_{x}^{2}}, \\
\left\|D_{x}^{1-1 / p} G(t) F\right\|_{L_{x}^{p}\left(L_{T}^{2}\right)} & \leq C T^{1 / p}\|F\|_{L_{x}^{1}\left(L_{T}^{2}\right)}, \\
\left\|D_{x}^{1 / 2} G(t) F\right\|_{L_{T}^{\infty}\left(L_{x}^{2}\right)} & \leq C\|F\|_{L_{x}^{1}\left(L_{T}^{2}\right)} .
\end{aligned}
$$

Proof of Lemma 3.1. The case $p=\infty$ and (3.3) are given in [13]. By (3.1) and (3.3), it is easy to see that, for $\lambda \in \mathbf{R}$,

$$
\begin{aligned}
\left\|D_{x}^{i \lambda} V(t) \phi\right\|_{L_{x}^{2}\left(L_{T}^{2}\right)} & \leq T^{1 / 2}\|\phi\|_{L_{x}^{2}}, \\
\left\|D_{x}^{1 / 2+i \lambda} V(t) \phi\right\|_{L_{x}^{\infty}\left(L_{T}^{2}\right)} & \leq C\|\phi\|_{L_{x}^{2}}, \\
\left\|D^{1 / 2+i \lambda} G(t) F\right\|_{L_{x}^{2}\left(L_{T}^{2}\right)} & \leq C T^{1 / 2}\|F\|_{L_{x}^{1}\left(L_{T}^{2}\right)} .
\end{aligned}
$$

where $C>0$ is independent of $\lambda$. Also, in [13], the following estimate appears:

$$
\left\|D_{x}^{1+i \lambda} G(t) F\right\|_{L_{x}^{\infty}\left(L_{T}^{2}\right)} \leq C\langle\lambda\rangle^{N}\|F\|_{L_{x}^{1}\left(L_{T}^{2}\right)},
$$

where $N$ is a large positive integer. Then, applying Stein's interpolation for analytic families of operators to the pairs (3.4)-(3.5) and (3.6)-(3.7), we obtain the desired estimates for $p \neq \infty$.

We next state the Strichartz estimates (for the proof, see [6, p. 377] and refer to [25]). These inequalities will be used for the weighted norm estimates.

Lemma 3.2. Let $p_{j}$ and $r_{j}(j=1,2)$ satisfy $0 \leq 2 / r_{j}=1 / 2-1 / p_{j} \leq$ $1 / 2$. Then, we have

$$
\begin{aligned}
&\|V(t) \phi\|_{L_{T}^{r_{1}}\left(L_{x}^{p_{1}}\right)} \leq C\|\phi\|_{L_{x}^{2}}, \\
&\|G(t) F\|_{L_{T}^{r_{1}}\left(L_{x}^{p_{1}}\right)} \leq C\|F\|_{L_{T}^{r_{2}^{\prime}}\left(L_{x}^{p_{2}^{\prime}}\right)},
\end{aligned}
$$

where $1 / p_{2}+1 / p_{2}^{\prime}=1 / r_{2}+1 / r_{2}^{\prime}=1$. 
We call $\|u(\cdot, x)\|_{L_{T}^{\infty}}$ the maximal function of $u$. Concerning the estimates of maximal functions, we have the following.

Lemma 3.3. Let $\sigma>1 / 2$ and $T \in[0,1]$. Then, we have

$$
\begin{aligned}
\|V(t) \phi\|_{L_{x}^{2}\left(L_{T}^{\infty}\right)} & \leq C\|\phi\|_{H_{x}^{\sigma, 0}} \\
\|G(t) F\|_{L_{x}^{2}\left(L_{T}^{\infty}\right)} & \leq C\|F\|_{L_{T}^{1}\left(H_{x}^{\sigma, 0}\right)} \\
\|G(t) F\|_{L_{x}^{2}\left(L_{T}^{\infty}\right)} & \leq C\left\|\left\langle D_{x}\right\rangle^{\sigma} F\right\|_{L_{T}^{1}\left(L_{x}^{1}\right)}
\end{aligned}
$$

Proof of Lemma 3.3. The estimate (3.10) is due to Vega [24]. From Minkowski's inequality, (3.11) follows. To prove (3.12), we first show that the integral kernel of $\left\langle D_{x}\right\rangle^{-\sigma} \exp \left(t \mathcal{H}_{x} \partial_{x}^{2}\right)$ (denoted by $K(t, x-y)$ ) is estimated as

$$
|K(t, x-y)| \leq C\left\{\begin{array}{l}
|x-y|^{-\sigma} \quad \text { if }|x-y|>1 \\
|x-y|^{-1+\sigma} \text { if }|x-y| \leq 1
\end{array}\right.
$$

where $C>0$ does not depend on $t \in(0, T]$. Let $z=x-y$ and write

$$
\begin{aligned}
K(t, z)= & (2 \pi)^{-1} \int_{-\infty}^{\infty} \exp (-i t \xi|\xi|+i z \xi)\langle\xi\rangle^{-\sigma} d \xi \\
= & (2 \pi)^{-1} \int_{0}^{\infty} \exp \left(-i t \xi^{2}+i z \xi\right)\langle\xi\rangle^{-\sigma} d \xi \\
& +(2 \pi)^{-1} \int_{-\infty}^{0} \exp \left(i t \xi^{2}+i z \xi\right)\langle\xi\rangle^{-\sigma} d \xi \\
\equiv & K_{+}(t, z)+K_{-}(t, z) .
\end{aligned}
$$

We only consider the estimate of $K_{+}(t, z)$ since $K_{-}(t, z)$ is similarly estimated. Changing the integral variable, we can write

$$
K_{+}(t, z)=\left\{\begin{array}{l}
e^{i z^{2} / 4 t} z^{\prime} \int_{-1}^{\infty} e^{-i t z^{\prime 2} \xi^{2}}\left\langle z^{\prime}(\xi+1)\right\rangle^{-\sigma} d \xi \quad \text { if } z>0, \\
-e^{i z^{2} / 4 t} z^{\prime} \int_{-\infty}^{-1} e^{-i t z^{\prime 2} \xi^{2}}\left\langle z^{\prime}(\xi+1)\right\rangle^{-\sigma} d \xi \text { if } z<0
\end{array}\right.
$$

where $z^{\prime}=z / 2 t$. Let us mainly consider the case $z>0$ step by step.

(The case $z>1$ ) The identity $\partial_{\xi} \xi e^{-i t z^{\prime 2} \xi^{2}}=\left(1-2 i t z^{\prime 2} \xi^{2}\right) e^{-i t z^{\prime 2} \xi^{2}}$ and integration by parts give

$$
\begin{aligned}
\left|K_{+}(t, z)\right| & \leq z^{\prime}\left|1-2 i t z^{\prime 2}\right|^{-1}+z^{\prime} \int_{-1}^{\infty}\left|\xi \partial_{\xi}\left(\left(1-2 i t z^{\prime 2} \xi^{2}\right)^{-1}\left\langle z^{\prime}(\xi+1)\right\rangle^{-\sigma}\right)\right| d \xi \\
& \leq C z^{-\sigma} .
\end{aligned}
$$


(The case $0<z \leq 1$ and $t z^{\prime 2}>1$ ) Let $\chi_{-1} \in C_{0}^{\infty}(\mathbf{R})$ with $\chi_{-1}(\xi)=1$ near $\xi=-1$, and let $\tilde{\chi}_{-1}=1-\chi_{-1}$. Then, we see that

$$
\begin{aligned}
\left|K_{+}(t, z)\right| \leq & \left|z^{\prime} \int_{-1}^{\infty} e^{-i t z^{\prime 2} \xi^{2}} \chi_{-1}(\xi)\left\langle z^{\prime}(\xi+1)\right\rangle^{-\sigma} d \xi\right| \\
& +\left|z^{\prime} \int_{-1}^{\infty} e^{-i t z^{\prime 2} \xi^{2}} \tilde{\chi}_{-1}(\xi)\left\langle z^{\prime}(\xi+1)\right\rangle^{-\sigma} d \xi\right| \\
\equiv & \left|K_{+, 1}(t, z)\right|+\left|K_{+, 2}(t, z)\right| .
\end{aligned}
$$

To estimate $\left|K_{+, 1}(t, z)\right|$, we use the identity $\partial_{\xi}\left(e^{-i t z^{\prime 2} \xi^{2}}-e^{-i t z^{\prime 2}}\right)=$ $-2 i t{z^{\prime}}^{2} \xi e^{-i t z^{\prime 2} \xi^{2}}$ and integration by parts. This yields

$$
\begin{aligned}
\left|K_{+, 1}(t, z)\right| \leq & C t^{-1} z^{\prime-1}\left(\int_{-1}^{\infty}\left(\partial_{\xi} \xi^{-1} \chi_{-1}\right)\left\langle z^{\prime}(\xi+1)\right\rangle^{-\sigma} d \xi\right. \\
& \left.+\int_{-1}^{\infty} \xi^{-1} \chi_{-1}\left|e^{-i t z^{\prime 2} \xi^{2}}-e^{-i t z^{\prime 2}}\right| z^{\prime}\left\langle z^{\prime}(\xi+1)\right\rangle^{-\sigma-1} d \xi\right) \\
\leq & C t^{-1} z^{\prime-1-\sigma}+C t^{-1} z^{\prime-1-\sigma} \int_{-1}^{0}\left|e^{-i t z^{\prime 2} \xi^{2}}-e^{-i t z^{\prime 2}}\right|(\xi+1)^{-\sigma-1} d \xi .
\end{aligned}
$$

The integral in (3.14) is bounded by $C\left(t z^{\prime}\right)^{\sigma}$ since, for $0<R<1$ and $\sigma^{\prime}>\sigma$, we have

$$
\begin{aligned}
& \int_{-1}^{0}\left|e^{-i t z^{\prime 2} \xi^{2}}-e^{-i t z^{\prime 2}}\right|(\xi+1)^{-\sigma-1} d \xi \\
& \quad \leq C \int_{-1+R}^{0}(\xi+1)^{-\sigma-1} d \xi+C \int_{-1}^{-1+R}\left(t z^{\prime 2}\right)^{\sigma^{\prime}}(\xi+1)^{\sigma^{\prime}-\sigma-1} d \xi \\
& \quad \leq C\left(R^{-\sigma}+\left(t z^{\prime 2}\right)^{\sigma^{\prime}} R^{\sigma^{\prime}-\sigma}\right)
\end{aligned}
$$

with $R=\left(\sigma /\left(\sigma^{\prime}-\sigma\right)\right)^{1 / \sigma^{\prime}}\left(t z^{\prime 2}\right)^{-1}$. Thus, noting that $z>2 t^{1 / 2}$ in this case, we have $\left|K_{+, 1}(t, z)\right| \leq C z^{-1+\sigma}$. The estimate $\left|K_{+, 2}(t, z)\right| \leq C z^{-1+\sigma}$ follows from the identity $\partial_{\xi} \xi e^{-i t z^{\prime 2} \xi^{2}}=\left(1-2 i t z^{\prime 2} \xi^{2}\right) e^{-i t z^{\prime 2} \xi^{2}}$ and integration by parts.

( The case $0<z \leq 1$ and $t z^{\prime 2} \leq 1$ ) Changing the integral variable, we have another expression of $K_{+}(t, z)$ such that

$$
\begin{aligned}
K_{+}(t, z)= & e^{i z^{2} / 4 t} t^{-1 / 2} \int_{-t^{1 / 2} z^{\prime}}^{\infty} e^{-i \xi^{2}}\left\langle t^{-1 / 2}\left(\xi+t^{1 / 2} z^{\prime}\right)\right\rangle^{-\sigma} d \xi \\
= & e^{i z^{2} / 4 t} t^{-1 / 2} \int_{-t^{1 / 2} z^{\prime}}^{\infty} e^{-i \xi^{2}} \chi_{0}(\xi)\left\langle t^{-1 / 2}\left(\xi+t^{1 / 2} z^{\prime}\right)\right\rangle^{-\sigma} d \xi \\
& +e^{i z^{2} / 4 t} t^{-1 / 2} \int_{-t^{1 / 2} z^{\prime}}^{\infty} e^{-i \xi^{2}}\left(1-\chi_{0}(\xi)\right)\left\langle t^{-1 / 2}\left(\xi+t^{1 / 2} z^{\prime}\right)\right\rangle^{-\sigma} d \xi \\
\equiv & K_{+, 3}(t, z)+K_{+, 4}(t, z)
\end{aligned}
$$


where $\chi_{0} \in C_{0}^{\infty}(\mathbf{R})$ with $\chi_{0}(\xi)=1$ in the neighborhood of $[-1,0]$. It is easy to see that $\left|K_{+, 3}(t, z)\right| \leq C t^{-(1-\sigma) / 2} \leq C z^{-1+\sigma}$. Making use of the identity $\partial_{\xi} e^{-i \xi^{2}}=-2 i \xi e^{-2 i \xi^{2}}$ and integration by parts, we can show that $\left|K_{+, 4}(t, z)\right| \leq C t^{-(1-\sigma) / 2} \leq C z^{-1+\sigma}$. Thus, (3.13) follows. Hence, (3.13) and Young's inequality yield (3.12).

In the nonlinear estimates, the fractional order differentiation will be applied to the quadratic term in (2.3). To handle this, we require the Leibnitz' type rule for the fractional order derivatives due to Kenig-Ponce-Vega [12, Appendix].

Lemma 3.4. Let $\sigma \in(0,1)$ and $\sigma_{0}, \sigma_{1} \in[0, \sigma]$ with $\sigma=\sigma_{0}+\sigma_{1}$. Also, let $q \in[1, \infty)$ and $q_{0}, q_{1}, r_{0}, r_{1} \in(1, \infty)$ with $1 / q=1 / q_{0}+1 / q_{1}$ and $1 / 2=$ $1 / r_{0}+1 / r_{1}$. Then, we have

$$
\left\|D_{x}^{\sigma}(f g)-\left(D_{x}^{\sigma} f\right) g-f\left(D_{x}^{\sigma} g\right)\right\|_{L_{x}^{q}\left(L_{T}^{2}\right)} \leq C\left\|D_{x}^{\sigma_{1}} f\right\|_{L_{x}^{q_{0}}\left(L_{T}^{r_{0}}\right)}\left\|D_{x}^{\sigma_{2}} g\right\|_{L_{x}^{q_{1}}\left(L_{T}^{r_{1}}\right)} .
$$

When we apply the Leibnitz' rule for the fractional order derivative to the nonlinearity, we encounter the estimate of lower order derivatives like $D_{x}^{s-1 / 2} u$ and $\partial_{x} u$. The following lemma and its corollary help us control these quantities. In particular, we require the case $q_{0}=1, q_{1}=\infty, r_{0}=\infty$ and $r_{1}=2$ (the end point case of the interpolation).

Lemma 3.5. Let $\sigma_{0}, \sigma_{1}>0, \alpha_{0}, \alpha_{1} \in \mathbf{R}$ and $q_{0}, q_{1}, r_{0}, r_{1} \in[1, \infty]$. Also, let $\sigma=(1-\theta) \sigma_{0}+\theta \sigma_{1}, \alpha=(1-\theta) \alpha_{0}+\theta \alpha_{1}, 1 / q=(1-\theta) / q_{0}+\theta / q_{1}$ and $1 / r=(1-\theta) / r_{0}+\theta / r_{1}$ with $\theta \in[0,1]$. Then, for $f \in \mathcal{S}\left(\mathbf{R} ; C^{\infty}[0, T]\right)$, we have

$$
\begin{aligned}
& \left\|D_{x}^{\sigma}\langle x\rangle^{\alpha} f\right\|_{L_{x}^{q}\left(L_{T}^{r}\right)} \\
& \leq\left(\sup _{\lambda \in \mathbf{R}} e^{-\lambda^{2}}\left\|D_{x}^{\sigma_{0}+i \lambda\left(\sigma_{1}-\sigma_{0}\right)}\langle x\rangle^{\alpha_{0}+i \lambda\left(\alpha_{1}-\alpha_{0}\right)} f\right\|_{L_{x}^{q_{0}}\left(L_{T}^{r_{0}}\right)}\right)^{1-\theta} \\
& \quad \times\left(\sup _{\lambda \in \mathbf{R}} e^{1-\lambda^{2}}\left\|D_{x}^{\sigma_{1}+i \lambda\left(\sigma_{1}-\sigma_{0}\right)}\langle x\rangle^{\alpha_{1}+i \lambda\left(\alpha_{1}-\alpha_{0}\right)} f\right\|_{L_{x}^{q_{1}}\left(L_{T}^{r_{1}}\right)}\right)^{\theta} .
\end{aligned}
$$

Proof of Lemma 3.5. We first define the complex valued function $F(z)$ by

$$
F(z)=e^{z^{2}} \int_{\mathbf{R} \times[0, T]} g_{z}(t, x) D_{x}^{\sigma(z)}\langle x\rangle^{\alpha(z)} f(t, x) d t d x,
$$

where $\sigma(z)=(1-z) \sigma_{0}+z \sigma_{1}, \alpha(z)=(1-z) \alpha_{0}+z \alpha_{1}$ and

$$
g_{z}(t, x)=\|g(\cdot, x)\|_{L_{T}^{r^{\prime}}}^{q^{\prime} / q^{\prime}(z)-r^{\prime} / r^{\prime}(z)}|g(t, x)|^{r^{\prime} / r^{\prime}(z)} \operatorname{sgn} g(t, x)
$$


with $g \in \mathcal{S}\left(\mathbf{R} ; C^{\infty}[0, T]\right), 1 / q^{\prime}(z)=(1-z) / q_{0}^{\prime}+z / q_{1}^{\prime}$ and $1 / r^{\prime}(z)=(1-z) / r_{0}^{\prime}+$ $z / r_{1}^{\prime}$ (the prime' denotes the Hölder conjugate). Then, $F(z)$ is holomorphic in the strip $S=\{z \in \mathbf{C} ; 0<\operatorname{Re} z<1\}$ and continuous in $\bar{S}$. In addition, $\lim _{|\operatorname{Im} z| \rightarrow \infty}|F(z)|=0$ in virtue of the multiplication $e^{z^{2}}$. According to the three line theorem, we see that

$$
|F(z)| \leq M_{0}^{1-\operatorname{Re} z} M_{1}^{\operatorname{Re} z},
$$

where $M_{j}=\sup _{\lambda \in \mathbf{R}}|F(j+i \lambda)|(j=0,1)$. By applying Hölder's inequality,

$$
M_{j} \leq\|g\|_{L_{x}^{q^{\prime}}\left(L_{T}^{r^{\prime}}\right)}^{q^{\prime} / q_{j}^{\prime}} \sup _{\lambda}\left(e^{j-\lambda^{2}}\left\|D_{x}^{\sigma_{j}+i \lambda\left(\sigma_{1}-\sigma_{0}\right)}\langle x\rangle^{\alpha_{j}+i \lambda\left(\alpha_{1}-\alpha_{0}\right)} f\right\|_{L_{x}^{q_{j}}\left(L_{T}^{r_{j}}\right)}\right) .
$$

Combining (3.17)-(3.18) and $\left(L_{x}^{q^{\prime}}\left(L_{T}^{r^{\prime}}\right)\right)^{\prime} \sim L_{x}^{q}\left(L_{T}^{r}\right)$ with $z=\theta$, we obtain Lemma 3.5.

Corollary 3.6. In addition to the assumptions in Lemma 3.5, let $\mu>0$. Then, we have

$$
\begin{aligned}
& \left\|\left\langle D_{x}\right\rangle^{\sigma}\langle x\rangle^{\alpha} f\right\|_{L_{x}^{q}\left(L_{T}^{r}\right)} \\
& \quad \leq C\left\|\left\langle D_{x}\right\rangle^{\sigma_{0}+\mu}\langle x\rangle^{\alpha_{0}} f\right\|_{L_{x}^{q_{0}}\left(L_{T}^{r_{0}}\right)}^{1-\theta}\left\|\left\langle D_{x}\right\rangle^{\sigma_{1}+\mu}\langle x\rangle^{\alpha_{1}} f\right\|_{L_{x}^{q_{1}}\left(L_{T}^{r_{1}}\right)}^{\theta} .
\end{aligned}
$$

Proof of Corollary 3.6. By estimating the integral kernels of operators, we see, for instance, that

$$
\left\|D_{x}^{\sigma_{0}+i \lambda\left(\sigma_{1}-\sigma_{0}\right)}\langle x\rangle^{i \lambda\left(\alpha_{1}-\alpha_{0}\right)}\left\langle D_{x}\right\rangle^{-\left(\sigma_{0}+\mu\right)}\right\|_{\mathcal{B}\left(L_{x}^{q_{0}}\left(L_{T}^{r_{0}}\right)\right)} \leq C\langle\lambda\rangle^{N}
$$

with $N$ sufficiently large. Then, Lemma 3.5 yields the desired result.

In our argument, the pseudo-differential operator $K_{\nu}$ often appears. We note that $K_{\nu} \notin \mathcal{B}\left(L_{x}^{1}\right)$ uniformly in $\nu \in(0,1]$ since the symbol of $K_{\nu}$ contains the gap for $\xi= \pm \infty$ if $\nu=0$. The following lemma states that $K_{\nu} \in \mathcal{B}\left(L_{x}^{p}\right)$ $(1<p<\infty)$ and its operator norm is estimated in terms of $\|\varphi\|_{X^{s}}$.

Lemma 3.7. Let $p \in(1, \infty)$ and $\nu \in(0,1)$. Then, we have

$$
\left\|K_{\nu}\right\|_{\mathcal{B}\left(L_{x}^{p}\right)} \leq C,
$$

where the positive constant $C$ is independent of $\nu \in(0,1]$ and does not diverge as $\varphi \rightarrow u_{0}$ in $X^{s}$. Furthermore, in the above inequalities, we may replace $L_{x}^{p}$ by $L_{x}^{p}\left(L_{T}^{r}\right)$ with $r \in(1, \infty)$. 
Proof of Lemma 3.7. Note that $K_{\nu}(x, \xi)=L_{\nu,+}(x, \xi) \chi_{+}(\xi)+L_{\nu,-} \chi_{-}(\xi)$, where

$$
L_{\nu, \pm}(x, \xi)=\exp \left( \pm i \sqrt{\frac{\pi}{2}}(1-\psi(\xi)) \hat{\eta}(\nu \xi) \int_{-\infty}^{x} \varphi d y\right)
$$

and $\chi_{+}(\xi)$ (resp. $\left.\chi_{-}(\xi)\right)$ is the characteristic function on $(0, \infty)$ (resp. $(-\infty, 0))$. It is well-known that $\chi_{ \pm}\left(i^{-1} \partial_{x}\right) \in \mathcal{B}\left(L_{x}^{p}\right)$, and thus it suffices to show that $L_{\nu, \pm}\left(x, i^{-1} \partial_{x}\right) \in \mathcal{B}\left(L_{x}^{p}\right)$. We write

$$
\begin{aligned}
L_{\nu, \pm}(x, \xi) & =\psi(\xi / 2) L_{\nu, \pm}(x, \xi)+(1-\psi(\xi / 2)) L_{\nu, \pm}(x, \xi) \\
& \equiv L_{\nu, \pm, 1}(x, \xi)+L_{\nu, \pm, 2}(x, \xi)
\end{aligned}
$$

By the integration by parts, the integral kernels of $L_{\nu, \pm, 1}\left(x, i^{-1} \partial_{x}\right)$ (denoted by $\left.L_{\nu, \pm, 1}[x, y]\right)$ are estimated as

$$
\begin{aligned}
\left|L_{\nu, \pm, 1}[x, y]\right| & =(2 \pi)^{-1}\left|\int e^{i(x-y) \xi} L_{\nu, \pm, 1}(x, \xi) d \xi\right| \\
& \leq C \exp \left(C\|\varphi\|_{X^{s}}\right)\langle x-y\rangle^{-N}
\end{aligned}
$$

where $N>0$ is sufficiently large. Also, note that

$$
\begin{aligned}
L_{\nu, \pm, 2}(x, \xi) \\
=(1-\psi(\xi / 2)) \exp \left( \pm i(\pi / 2)^{1 / 2}(1-\psi(\xi)) \hat{\eta}(\nu \xi) \int_{-\infty}^{x} \varphi(y) d y\right) \\
=(1-\psi(\xi / 2)) \\
\quad+(1-\psi(\xi / 2))\left(\exp \left( \pm i(\pi / 2)^{1 / 2} \hat{\eta}(\nu \xi) \int_{-\infty}^{x} \varphi(y) d y\right)-1\right)
\end{aligned}
$$

where we remarked that, if $1-\psi(\xi / 2) \neq 0$, then $1-\psi(\xi)=1$. Furthermore, the symbol $\exp \left(i^{-1}(\pi / 2)^{1 / 2} \hat{\eta}(\nu \xi) \int_{-\infty}^{x} \varphi(y) d y\right)-1$ yields the integral operator with the kernel bounded by $C \exp \left(C\|\varphi\|_{X^{s}}\right) \nu^{-1}\langle(x-y) / \nu\rangle^{-N}$, and $1-\psi(\xi / 2)$ yields $L_{x}^{p}$-bounded operator. Hence, we see that $L_{\nu, \pm, 2}\left(x, i^{-1} \partial_{x}\right) \in \mathcal{B}\left(L_{x}^{p}\right)$ and (3.20) follows.

\section{$\S 4 . \quad$ Weighted Norm Estimates}

In this section, we derive several linear estimates in the weighted norms, which bring us the persistence of the solution. 
Lemma 4.1. Let $\sigma, \alpha \in[1 / 2,1)$ and $\sigma^{\prime}>\sigma+\alpha$. Then, for $T \in(0,1)$, we have

$$
\begin{aligned}
\|V(t) \phi\|_{L_{T}^{\infty}\left(H_{x}^{\sigma, \alpha}\right) \leq} & C\left(\|\phi\|_{H_{x}^{\sigma^{\prime}, 0}}+\|\phi\|_{H_{x}^{\sigma, \alpha}}\right) \\
\|G(t) F\|_{L_{T}^{\infty}\left(H_{x}^{\sigma, \alpha}\right) \leq} & C T^{1 / 2}\left\|D_{x}^{\sigma^{\prime}-1 / 2} F\right\|_{L_{x}^{1}\left(L_{T}^{2}\right)} \\
& +C\left\|\left\langle D_{x}\right\rangle^{\sigma}\langle x\rangle^{\alpha} F\right\|_{L_{T}^{1}\left(L_{x}^{2}\right)+L_{T}^{4 / 3}\left(L_{x}^{1}\right)^{2}}
\end{aligned}
$$

Proof of Lemma 4.1. Let $w=V(t) \phi$ and multyply $\langle x\rangle^{\alpha}\left\langle D_{x}\right\rangle^{\sigma}$ on both hand sides of $\left(\partial_{t}+\mathcal{H}_{x} \partial_{x}^{2}\right) w=0$ with $w(0, x)=\phi$, we have

$$
\left(\partial_{t}+\mathcal{H}_{x} \partial_{x}^{2}\right)\langle x\rangle^{\alpha}\left\langle D_{x}\right\rangle^{\sigma} w=\left[\mathcal{H}_{x} \partial_{x}^{2},\langle x\rangle^{\alpha}\right]\left\langle D_{x}\right\rangle^{\sigma} w .
$$

Thus, Duhamel's principle gives

$$
\langle x\rangle^{\alpha}\left\langle D_{x}\right\rangle^{\sigma} w=V(t)\langle x\rangle^{\alpha}\left\langle D_{x}\right\rangle^{\sigma} \phi+G(t)\left[\mathcal{H}_{x} \partial_{x}^{2},\langle x\rangle^{\alpha}\right]\left\langle D_{x}\right\rangle^{\sigma} w .
$$

Note that the symbolic calculation of the pseudo-differential operators gives

$$
\left[\mathcal{H}_{x} \partial_{x}^{2},\langle x\rangle^{\alpha}\right]=2\left(\partial_{x}\langle x\rangle^{\alpha}\right)\left(1-\psi\left(i^{-1} \partial_{x}\right)\right) D_{x}+\left(\mathcal{B}\left(L^{2}\right) \text { operator }\right),
$$

where $\psi\left(i^{-1} \partial_{x}\right)=\mathcal{F}^{-1} \psi(\xi) \mathcal{F}$ where $\psi(\xi)$ is defined by $(2.2)$. Then, applying Lemma 3.2 and Hölder's inequality to (4.3), we have

$$
\|V(t) \phi\|_{L_{T}^{\infty}\left(H_{x}^{\sigma, \alpha}\right)} \leq C\|\phi\|_{H_{x}^{\sigma, \alpha}}+C T^{1 / 2}\left\|\left(1-\psi\left(i^{-1} \partial_{x}\right)\right) D_{x} V(t)\left\langle D_{x}\right\rangle^{\sigma} \phi\right\|_{L_{x}^{q}\left(L_{T}^{2}\right)},
$$

where $1 / q>\alpha-1 / 2$. Also, Lemma 3.1 (3.1) gives

$$
\begin{aligned}
\|V(t) \phi\|_{L_{T}^{\infty}\left(H_{x}^{\sigma, \alpha}\right)} & \leq C\|\phi\|_{H_{x}^{\sigma, \alpha}}+C T^{1 / 2}\left\|D_{x}^{1 / q+1 / 2}\left\langle D_{x}\right\rangle^{\sigma} \phi\right\|_{L_{x}^{2}} \\
& \leq C\left(\|\phi\|_{H_{x}^{\sigma, \alpha}}+\|\phi\|_{H_{x}^{\sigma^{\prime}, 0}}\right) .
\end{aligned}
$$

We next prove (4.2). Similarly to the derivation of (4.3), we have

$$
\begin{aligned}
\langle x\rangle^{\alpha}\left\langle D_{x}\right\rangle^{\sigma} G(t) F & =G(t)\langle x\rangle^{\alpha}\left\langle D_{x}\right\rangle^{\sigma} F+G(t)\left[\mathcal{H}_{x} \partial_{x}^{2},\langle x\rangle^{\alpha}\right] G(t)\left\langle D_{x}\right\rangle^{\sigma} F \\
& \equiv I_{1}+I_{2} .
\end{aligned}
$$

By Lemma $3.2(3.9), I_{1}$ is estimated as

$$
\left\|I_{1}\right\|_{L_{T}^{\infty}\left(L_{x}^{2}\right)} \leq C\left\|\langle x\rangle^{\alpha}\left\langle D_{x}\right\rangle^{\sigma} F\right\|_{L_{T}^{1}\left(L_{x}^{2}\right)+L_{T}^{4 / 3}\left(L_{x}^{1}\right)} .
$$

As for $I_{2}$, we see that

$$
\begin{aligned}
&\left\|I_{2}\right\|_{L_{T}^{\infty}\left(L_{x}^{2}\right)} \\
& \leq C\left\|\left(\partial_{x}\langle x\rangle^{\alpha}\right)\left(1-\psi\left(i^{-1} \partial_{x}\right)\right) D_{x} G(t)\left\langle D_{x}\right\rangle^{\sigma} F\right\|_{L_{T}^{1}\left(L_{x}^{2}\right)} \\
&+C\left\|G(t)\left\langle D_{x}\right\rangle^{\sigma} F\right\|_{L_{T}^{1}\left(L_{x}^{2}\right)} \\
& \leq C T^{1 / 2}\left\|D_{x} G(t)\left\langle D_{x}\right\rangle^{\sigma} F\right\|_{L_{x}^{q}\left(L_{T}^{2}\right)}+C\left\|\langle x\rangle^{\alpha}\left\langle D_{x}\right\rangle^{\sigma} F\right\|_{L_{T}^{1}\left(L_{x}^{2}\right)+L_{T}^{4 / 3}\left(L_{x}^{1}\right)}
\end{aligned}
$$


Note that, to obtain the last inequality of (4.5), we used $1-\psi\left(i^{-1} \partial_{x}\right) \in$ $\mathcal{B}\left(L_{x}^{q}\left(L_{T}^{2}\right)\right)$ and Lemma $3.2(3.9)$. Lemmas 3.1, 3.2 and $D_{x}\left(\left\langle D_{x}\right\rangle^{\sigma}-D_{x}^{\sigma}\right) \in$ $\mathcal{B}\left(L^{p}\right)(1 \leq p \leq \infty)$ yield

$$
\begin{gathered}
\left\|D_{x} G(t)\left\langle D_{x}\right\rangle^{\sigma} F\right\|_{L_{x}^{q}\left(L_{T}^{2}\right)} \leq C\left\|D_{x}^{1 / q+\sigma} F\right\|_{L_{x}^{1}\left(L_{T}^{2}\right)}+C\|F\|_{L_{T}^{1}\left(L_{x}^{2}\right)+L_{T}^{4 / 3}\left(L_{x}^{1}\right)} \\
\leq C\left\|D_{x}^{\sigma^{\prime}-1 / 2} F\right\|_{L_{x}^{1}\left(L_{T}^{2}\right)}+C\left\|\langle x\rangle^{\alpha}\left\langle D_{x}\right\rangle^{\sigma} F\right\|_{L_{T}^{1}\left(L_{x}^{2}\right)+L_{T}^{4 / 3}\left(L_{x}^{1}\right)^{\prime}}
\end{gathered}
$$

Hence, Lemma 4.1 follows.

The lemma given below is concerning the estimates of maximal function and determines the regularity and weight conditions on the initial data.

Lemma 4.2. Let $\mu \in[0,1), \alpha \in[1 / 2,1), \sigma>\mu+1 / 2$ and $\sigma^{\prime}>\mu+\alpha+$ $1 / 2$. Then, for $T \in(0,1)$, we have

$$
\begin{aligned}
\left\|\left\langle D_{x}\right\rangle^{\mu}\langle x\rangle^{\alpha} V(t) \phi\right\|_{L_{x}^{2}\left(L_{T}^{\infty}\right)} \leq & C\left(\|\phi\|_{H_{x}^{\sigma^{\prime}, 0}}+\|\phi\|_{H_{x}^{\sigma, \alpha}}\right) \\
\left\|\left\langle D_{x}\right\rangle^{\mu}\langle x\rangle^{\alpha} G(t) F\right\|_{L_{x}^{2}\left(L_{T}^{\infty}\right) \leq} & C T^{1 / 2}\left\|D_{x}^{\sigma^{\prime}-1 / 2} F\right\|_{L_{x}^{1}\left(L_{T}^{2}\right)} \\
& +C\left\|\left\langle D_{x}\right\rangle^{\sigma}\langle x\rangle^{\alpha} F\right\|_{L_{T}^{1}\left(L_{x}^{2}\right)+L_{T}^{4 / 3}\left(L_{x}^{1}\right)}
\end{aligned}
$$

Proof of Lemma 4.2. We only prove (4.7) since (4.6) follows more easily. Applying Lemmas 3.2 and 3.3 to (4.4), we have

$$
\begin{aligned}
&\left\|\left\langle D_{x}\right\rangle^{\mu}\langle x\rangle^{\alpha} G(t) F\right\|_{L_{x}^{2}\left(L_{T}^{\infty}\right)} \\
& \leq\left\|\left\langle D_{x}\right\rangle^{\mu} G(t)\langle x\rangle^{\alpha} F\right\|_{L_{x}^{2}\left(L_{T}^{\infty}\right)}+\left\|\left\langle D_{x}\right\rangle^{\mu} G(t)\left[\mathcal{H}_{x} \partial_{x}^{2},\langle x\rangle^{\alpha}\right] G(\cdot) F\right\|_{L_{x}^{2}\left(L_{T}^{\infty}\right)} \\
& \leq C\left\|\left\langle D_{x}\right\rangle^{\sigma}\langle x\rangle^{\alpha} F\right\|_{L_{T}^{1}\left(L_{T}^{2}\right)+L_{T}^{1}\left(L_{x}^{1}\right)}+C T\left\|\left\langle D_{x}\right\rangle^{\mu+1 / 2+\epsilon} P_{1} G(t) F\right\|_{L_{T}^{\infty}\left(L_{x}^{2}\right)} \\
&+C T^{1 / 2}\left\|\left\langle D_{x}\right\rangle^{\mu+1 / 2+\epsilon} P_{2} G(t) F\right\|_{L_{x}^{2}\left(L_{T}^{2}\right)},
\end{aligned}
$$

where $\mu+1 / 2+\epsilon<\sigma, P_{1}=\left[\psi\left(i^{-1} \partial_{x}\right) \mathcal{H}_{x} \partial_{x}^{2},\langle x\rangle^{\alpha}\right]$ and $P_{2}=\left[\left(1-\psi\left(i^{-1}\right)\right) \mathcal{H}_{x} \partial_{x}^{2}\right.$, $\left.\langle x\rangle^{\alpha}\right]$ with $\psi \in C_{0}^{\infty}(\mathbf{R})$. By Hölder's inequality, the first term on the right hand side of (4.8) is estimated as

$$
\left\|\left\langle D_{x}\right\rangle^{\sigma}\langle x\rangle^{\alpha} F\right\|_{L_{T}^{1}\left(L_{x}^{2}\right)+L_{T}^{1}\left(L_{x}^{1}\right)} \leq C\left\|\left\langle D_{x}\right\rangle^{\sigma}\langle x\rangle^{\alpha} F\right\|_{L_{T}^{1}\left(L_{x}^{2}\right)+L_{T}^{4 / 3}\left(L_{x}^{1}\right)} .
$$

Since $P_{1} f=\int P_{1}(x, y) f(y) d y$, where

$$
P_{1}(x, y)=-\left.(2 \pi)^{-1} \int e^{i(x-y) \xi} \partial_{\xi}(\psi(\xi) \xi|\xi|) d \xi \int_{0}^{1} \partial_{w}\langle w\rangle^{\alpha}\right|_{w=\theta y+(1-\theta) x} d \theta
$$


and $\left|\partial_{x}^{k} P_{1}(x, y)\right| \leq C\langle x-y\rangle^{-2}(k=0,1,2)$, the second term in (4.8) is estimated as

$$
\begin{aligned}
\left\|\left\langle D_{x}\right\rangle^{\mu+1 / 2+\epsilon} P_{1} G(t) F\right\|_{L_{T}^{\infty}\left(L_{x}^{2}\right)} & \leq C\|G(t) F\|_{L_{T}^{\infty}\left(L_{x}^{2}\right)} \\
& \leq C\|F\|_{L_{T}^{1}\left(L_{x}^{2}\right)+L_{T}^{4 / 3}\left(L_{x}^{1}\right)} \quad \text { (by Lemma 3.2) } \\
& \leq C\left\|\left\langle D_{x}\right\rangle^{\sigma}\langle x\rangle^{\alpha} F\right\|_{L_{T}^{1}\left(L_{x}^{2}\right)+L_{T}^{4 / 3}\left(L_{x}^{1}\right)}
\end{aligned}
$$

As for the third term in (4.8), we note that

$$
\begin{aligned}
P_{2}= & 2 \partial_{x}\langle x\rangle^{\alpha}\left(1-\psi\left(i^{-1} \partial_{x}\right)\right) D_{x} \\
& +\left(\text { pseudo-differential operator with symbol in } S^{0}\right) .
\end{aligned}
$$

Then, it follows that

$$
\begin{aligned}
& \left\|\left\langle D_{x}\right\rangle^{\mu+1 / 2+\epsilon} P_{2} G(t) F\right\|_{L_{x}^{2}\left(L_{T}^{2}\right)} \\
& \leq C\left\|\left\langle D_{x}\right\rangle^{\mu+1 / 2+\epsilon}\left(\partial_{x}\langle x\rangle^{\alpha}\right)\left(1-\psi\left(i^{-1} \partial_{x}\right)\right) D_{x} G(t) F\right\|_{L_{T}^{\infty}\left(L_{x}^{2}\right)} \\
& \quad+C T^{1 / 2}\left\|\left\langle D_{x}\right\rangle^{\mu+1 / 2+\epsilon} G(t) F\right\|_{L_{T}^{\infty}\left(L_{x}^{2}\right)} .
\end{aligned}
$$

Since the symbol of $\left[\left\langle D_{x}\right\rangle^{\mu+1 / 2+\epsilon}, \partial_{x}\langle x\rangle^{\alpha}\right]$ belongs to $S^{\mu-1 / 2+\epsilon}$, Lemmas 3.1 and 3.2 give

$$
\begin{aligned}
& \left\|\left\langle D_{x}\right\rangle^{\mu+1 / 2+\epsilon} P_{2} G(t) F\right\|_{L_{x}^{2}\left(L_{T}^{2}\right)} \\
& \quad \leq C\left\|D_{x}^{\mu+3 / 2+\epsilon} G F\right\|_{L_{x}^{q}\left(L_{T}^{2}\right)}+C T^{1 / 2}\left\|G\left\langle D_{x}\right\rangle^{\sigma} F\right\|_{L_{T}^{\infty}\left(L_{x}^{2}\right)} \\
& \quad \leq C\left\|D_{x}^{\sigma^{\prime}-1 / 2} F\right\|_{L_{x}^{1}\left(L_{T}^{2}\right)}+C\left\|\left\langle D_{x}\right\rangle^{\sigma}\langle x\rangle^{\alpha} F\right\|_{L_{T}^{1}\left(L_{x}^{2}\right)+L_{T}^{4 / 3}\left(L_{x}^{1}\right)}
\end{aligned}
$$

where $1 / q>\alpha-1 / 2$. Combining (4.8)-(4.11), we obtain Lemma 4.2.

\section{§5. Nonlinear Estimates}

In what follows, we only consider the case $s \in(1,3 / 2)$ since the other cases are verified without major modification. For a brief description, we let

$$
\begin{aligned}
\|u\|_{\text {initial }} & =\|u\|_{L_{T}^{\infty}\left(X^{s}\right)}, \\
\|u\|_{\text {smooth }} & =\left\|\langle x\rangle^{-\rho}\left\langle D_{x}\right\rangle^{s+1 / 2} u\right\|_{L_{x}^{1 / \varepsilon}\left(L_{T}^{2}\right)}, \\
\|u\|_{\text {maxim }} & =\left\|\left\langle D_{x}\right\rangle^{\mu}\langle x\rangle^{\alpha_{1}} u\right\|_{L_{x}^{2}\left(L_{T}^{\infty}\right)},
\end{aligned}
$$

and thus $\|u\|_{Y_{T}}$ (introduced in section 1) equals to $\|u\|_{\text {initial }}+\|u\|_{\text {smooth }}+$ $\|u\|_{\text {maxim }}$. 
Lemma 5.1. There exist some $C, C_{\varphi}, \beta>0$ and $\theta \in(0,1)$ such that

$$
\begin{aligned}
& \left\|\left\langle D_{x}\right\rangle^{s-1 / 2} K_{\nu}\left(f \partial_{x} g\right)\right\|_{L_{x}^{1}\left(L_{T}^{2}\right)} \\
& \leq C\|f\|_{\text {maxim }}\|g\|_{\text {smooth }}+C\|f\|_{\text {maxim }}^{\theta}\|f\|_{\text {smooth }}^{1-\theta}\|g\|_{\text {maxim }}^{1-\theta}\|g\|_{\text {smooth }}^{\theta} \\
& \quad+C_{\varphi} T^{\beta}\|f\|_{Y_{T}}\|g\|_{Y_{T}}, \\
& \left\|\left\langle D_{x}\right\rangle^{s_{1}}\langle x\rangle^{\alpha_{1}}\left(f \partial_{x} g\right)\right\|_{L_{T}^{1}\left(L_{x}^{2}\right)+L_{T}^{4 / 3}\left(L_{x}^{1}\right)} \leq C T^{\beta}\|f\|_{Y_{T}}\|g\|_{Y_{T}},
\end{aligned}
$$

where $C_{\varphi}$ may diverge as $\varphi \rightarrow u_{0}$ in $X^{s}$.

Proof of Lemma 5.1. For small $\kappa>0$, we write

$$
\begin{aligned}
\left\langle D_{x}\right\rangle^{s-1 / 2} K_{\nu}\left(f \partial_{x} g\right)= & \left\langle D_{x}\right\rangle^{s-1 / 2} K_{\nu}\langle x\rangle^{-\kappa}\left(\langle x\rangle^{\kappa} f \partial_{x} g\right) \\
= & \langle x\rangle^{-\kappa} K_{\nu}\left\langle D_{x}\right\rangle^{s-1 / 2}\left(\langle x\rangle^{\kappa} f \partial_{x} g\right) \\
& +\langle x\rangle^{-\kappa}\left[\left\langle D_{x}\right\rangle^{s-1 / 2}, K_{\nu}\right]\left(\langle x\rangle^{\kappa} f \partial_{x} g\right) \\
& +\left[\left\langle D_{x}\right\rangle^{s-1 / 2} K_{\nu},\langle x\rangle^{-\kappa}\right]\left(\langle x\rangle^{\kappa} f \partial_{x} g\right)
\end{aligned}
$$

Applying Hölder's inequality to the first term on the right hand side and noting that $\left[\left\langle D_{x}\right\rangle^{s-1 / 2}, K_{\nu}\right] \in \mathcal{B}\left(L_{x}^{1}\left(L_{T}^{2}\right)\right)$ and $\left[\left\langle D_{x}\right\rangle^{s-1 / 2} K_{\nu},\langle x\rangle^{-\kappa}\right] \in \mathcal{B}\left(L_{x}^{1}\left(L_{T}^{2}\right)\right)$ since the symbol of these commutators belong to $S^{s-3 / 2}$ with $s-3 / 2<0$, we have

$$
\begin{aligned}
\left\|\left\langle D_{x}\right\rangle^{s-1 / 2} K_{\nu}\left(f \partial_{x} g\right)\right\|_{L_{x}^{1}\left(L_{T}^{2}\right) \leq} & C\left\|K_{\nu}\left\langle D_{x}\right\rangle^{s-1 / 2}\left(\langle x\rangle^{\kappa} f \partial_{x} g\right)\right\|_{L_{x}^{p}\left(L_{T}^{2}\right)} \\
& +C_{\varphi}\left\|\langle x\rangle^{\kappa} f \partial_{x} g\right\|_{L_{x}^{1}\left(L_{T}^{2}\right)} \\
\equiv & I_{1}+I_{2}
\end{aligned}
$$

where $1<p<1 /(1-\kappa)$. As for $I_{2}$, the Hölder and Sobolev inequalities yield

$$
\begin{aligned}
I_{2} & \leq C_{\varphi}\left\|\langle x\rangle^{\alpha_{1}} f \partial_{x} g\right\|_{L_{x}^{2}\left(L_{T}^{2}\right)} \\
& \leq C_{\varphi} T^{1 / 2}\|f\|_{L_{T}^{\infty}\left(H_{x}^{s_{1}, \alpha_{1}}\right)}\|g\|_{L_{T}^{\infty}\left(H_{x}^{s, 0}\right)} .
\end{aligned}
$$

By Lemma 3.7 and $\left\langle D_{x}\right\rangle^{s-1 / 2}-D_{x}^{s-1 / 2} \in \mathcal{B}\left(L_{x}^{p}\left(L_{T}^{2}\right)\right)$, we see that

$$
\begin{aligned}
I_{1} & \leq C\left\|D_{x}^{s-1 / 2}\left(\langle x\rangle^{\kappa} f \partial_{x} g\right)\right\|_{L_{x}^{p}\left(L_{T}^{2}\right)}+C\left\|\langle x\rangle^{\kappa} f \partial_{x} g\right\|_{L_{x}^{p}\left(L_{T}^{2}\right)} \\
& \equiv I_{11}+I_{12} .
\end{aligned}
$$

$I_{12}$ is estimated as

$$
\begin{aligned}
I_{12} & \leq C\left\|\langle x\rangle^{\kappa+1 / 2} f \partial_{x} g\right\|_{L_{x}^{2}\left(L_{T}^{2}\right)} \\
& \leq C T^{1 / 2}\left\|\langle x\rangle^{\alpha_{1}} f\right\|_{L_{T}^{\infty}\left(L_{x}^{\infty}\right)}\|g\|_{L_{T}^{\infty}\left(H_{x}^{1,0}\right)} \\
& \leq C T^{1 / 2}\|f\|_{L_{T}^{\infty}\left(H_{x}^{s_{1}, \alpha_{1}}\right)}\|g\|_{L_{T}^{\infty}\left(H_{x}^{s, 0}\right)}
\end{aligned}
$$


since $\kappa+1 / 2<\alpha_{1}$. By Lemma 3.4, we have

$$
\begin{aligned}
I_{11} \leq & \left\|\langle x\rangle^{\kappa} f D_{x}^{s-1 / 2} \partial_{x} g\right\|_{L_{x}^{p}\left(L_{T}^{2}\right)} \\
& +C\left\|D_{x}^{s-1 / 2}\langle x\rangle^{\kappa} f\right\|_{L_{x}^{p_{1}}\left(L_{T}^{r_{1}}\right)}\left\|\partial_{x} g\right\|_{L_{x}^{p_{2}}\left(L_{T}^{r_{2}}\right)} \\
\equiv & I_{111}+I_{112},
\end{aligned}
$$

where

$$
\begin{aligned}
& 1 / p_{1}=\theta(1 / p-\varepsilon)+(1-\theta) \varepsilon, \\
& 1 / r_{1}=\theta / \infty+(1-\theta) / 2, \\
& 1 / p_{2}=(1-\theta)(1 / p-\varepsilon)+\theta \varepsilon, \\
& 1 / r_{2}=(1-\theta) / \infty+\theta / 2,
\end{aligned}
$$

with $\theta \mu / 2+(1-\theta)(s+1 / 2-\mu / 2)=s-1 / 2, \theta \in(0,1)$ and $\mu>0$ small enough. By Hölder's inequality and $\mathcal{H}_{x} \in \mathcal{B}\left(L_{x}^{1 / \varepsilon}\left(L_{T}^{2}\right)\right)$, we see that

$$
\begin{aligned}
I_{111} & \leq\left\|\langle x\rangle^{\kappa+\rho} f\right\|_{L_{x}^{p /(1-\varepsilon p)}\left(L_{T}^{\infty}\right)}\left\|\langle x\rangle^{-\rho} D_{x}^{s-1 / 2} \partial_{x} g\right\|_{L_{x}^{1 / \varepsilon}\left(L_{T}^{2}\right)} \\
& \leq C\|f\|_{\text {maxim }}\left(\|g\|_{\text {smooth }}+T^{1 / 2}\|g\|_{\text {initial }}\right) .
\end{aligned}
$$

To estimate $I_{112}$ by the interpolation (Corollary 3.6), we choose $\kappa^{\prime}>\kappa$ and $\rho^{\prime}>0$ so that

$$
\begin{aligned}
\kappa^{\prime} & =\theta \rho^{\prime}+(1-\theta)(-\rho), \\
0 & =(1-\theta) \rho^{\prime}+\theta(-\rho) .
\end{aligned}
$$

Then, we see that

$$
\begin{aligned}
& \left\|D_{x}^{s-1 / 2}\langle x\rangle^{\kappa} f\right\|_{L_{x}^{p_{1}}\left(L_{T}^{r_{1}}\right)} \\
& \leq\left\|\left\langle D_{x}\right\rangle^{s-1 / 2}\langle x\rangle^{\kappa^{\prime}}\langle x\rangle^{-\left(\kappa^{\prime}-\kappa\right)} f\right\|_{L_{x}^{p_{1}\left(L_{T}^{r_{1}}\right)}} \\
& \leq C\left\|\left\langle D_{x}\right\rangle^{\mu}\langle x\rangle^{\rho^{\prime}-\kappa^{\prime}+\kappa} f\right\|_{L_{x}^{p /(1-\varepsilon p)}\left(L_{T}^{\infty}\right)}^{\theta}\left\|\left\langle D_{x}\right\rangle^{s+1 / 2}\langle x\rangle^{-\rho-\kappa^{\prime}+\kappa} f\right\|_{L_{x}^{1 / \varepsilon}\left(L_{T}^{2}\right)}^{1-\theta} \\
& \leq C\left\|\langle x\rangle^{1 / 2}\left\langle D_{x}\right\rangle^{\mu}\langle x\rangle^{\rho^{\prime}-\kappa^{\prime}+\kappa} f\right\|_{L_{x}^{2}\left(L_{T}^{\infty}\right)}^{\theta}\left\|\left\langle D_{x}\right\rangle^{s+1 / 2}\langle x\rangle^{-\rho-\kappa^{\prime}+\kappa} f\right\|_{L_{x}^{1 / \varepsilon}\left(L_{T}^{2}\right)}^{1-\theta}
\end{aligned}
$$

We write

$$
\begin{aligned}
\langle x\rangle^{1 / 2}\left\langle D_{x}\right\rangle^{\mu}\langle x\rangle^{\rho^{\prime}-\kappa^{\prime}+\kappa} f= & \langle x\rangle^{\rho^{\prime}-\kappa^{\prime}+\kappa-\alpha_{1}+1 / 2}\left\langle D_{x}\right\rangle^{\mu}\langle x\rangle^{\alpha_{1}} f \\
& +\left[\left\langle D_{x}\right\rangle^{\mu},\langle x\rangle^{\rho^{\prime}-\kappa^{\prime}+\kappa-\alpha_{1}+1 / 2}\right]\langle x\rangle^{\alpha_{1}} f \\
& +\left[\langle x\rangle^{1 / 2},\left\langle D_{x}\right\rangle^{\mu}\right]\langle x\rangle^{\rho^{\prime}-\kappa^{\prime}+\kappa} f .
\end{aligned}
$$


Since $\rho$ and $\kappa$ are taken so small that $\rho^{\prime}-\kappa^{\prime}+\kappa-\alpha_{1}+1 / 2<0$ and all the commutators in the above are $L_{x}^{2}\left(L_{T}^{\infty}\right)$-bounded, we have

$$
\left\|\langle x\rangle^{1 / 2}\left\langle D_{x}\right\rangle^{\mu}\langle x\rangle^{\rho^{\prime}-\kappa^{\prime}+\kappa} f\right\|_{L_{x}^{2}\left(L_{T}^{\infty}\right)} \leq C\|f\|_{\text {maxim }} .
$$

On the other hand, we write

$$
\begin{aligned}
\left\langle D_{x}\right\rangle^{s+1 / 2}\langle x\rangle^{-\rho-\kappa^{\prime}+\kappa} f= & \langle x\rangle^{-\rho-\kappa^{\prime}+\kappa}\langle x\rangle^{s+1 / 2} f \\
& +\left[\left\langle D_{x}\right\rangle^{s+1 / 2},\langle x\rangle^{-\rho-\kappa^{\prime}+\kappa}\right] f .
\end{aligned}
$$

By $\sigma\left(\left[\left\langle D_{x}\right\rangle^{s+1 / 2},\langle x\rangle^{-\rho-\kappa^{\prime}+\kappa}\right]\right) \in S^{s-1 / 2}$ and the Sobolev embedding $H_{x}^{1 / 2,0} \subset$ $L_{x}^{1 / \varepsilon}$

$$
\begin{aligned}
\left\|\left\langle D_{x}\right\rangle^{s+1 / 2}\langle x\rangle^{-\rho-\kappa^{\prime}+\kappa} f\right\|_{L_{x}^{1 / \varepsilon}\left(L_{T}^{2}\right)} \leq & \left\|\langle x\rangle^{-\rho-\kappa^{\prime}+\kappa}\langle x\rangle^{s+1 / 2} f\right\|_{L_{x}^{1 / \varepsilon}\left(L_{T}^{2}\right)} \\
& +T^{1 / 2}\left\|\left[\left\langle D_{x}\right\rangle^{s+1 / 2},\langle x\rangle^{-\rho-\kappa^{\prime}+\kappa}\right] f\right\|_{L_{T}^{\infty}\left(H_{x}^{1 / 2,0}\right)} \\
\leq & C\left(\|f\|_{\text {smooth }}+T^{1 / 2}\|f\|_{\text {initial }}\right) .
\end{aligned}
$$

By (5.3)-(5.5), we see that

$$
\begin{aligned}
& \left\|D_{x}^{s-1 / 2}\langle x\rangle^{\kappa} f\right\|_{L_{x}^{p_{1}}\left(L_{T}^{r_{1}}\right)} \\
& \quad \leq C\|f\|_{\text {maxim }}^{\theta}\left(\|f\|_{\text {smooth }}+T^{1 / 2}\|f\|_{\text {initial }}\right)^{1-\theta} \\
& \quad \leq C\|f\|_{\text {maxim }}^{\theta}\|f\|_{\text {smooth }}^{1-\theta}+C T^{\beta}\|f\|_{Y_{T}} .
\end{aligned}
$$

As for $\left\|\partial_{x} g\right\|_{L_{x}^{p_{2}}\left(L_{T}^{r_{2}}\right)}$, we use $\mathcal{H}_{x} \in \mathcal{B}\left(L_{x}^{p_{2}}\left(L_{T}^{r_{2}}\right)\right)$ and Corollary 3.6. Then,

$$
\begin{aligned}
\left\|\partial_{x} g\right\|_{L_{x}^{p_{2}}\left(L_{T}^{r_{2}}\right)} & \leq C\left\|\left\langle D_{x}\right\rangle g\right\|_{L_{x}^{p_{2}}\left(L_{T}^{r_{2}}\right)} \\
& \leq C\|g\|_{\text {maxim }}^{1-\theta}\left(\|g\|_{\text {smooth }}+T^{1 / 2}\|g\|_{\text {initial }}\right)^{\theta} \\
& \leq C\|g\|_{\text {maxim }}^{1-\theta}\|g\|_{\text {smooth }}^{\theta}+C T^{\beta}\|g\|_{Y_{T} .} .
\end{aligned}
$$

Thus, combining (5.6) and (5.7), we obtan (5.1). We next prove (5.2). It suffices to estimate $\left\|D_{x}^{s_{1}}\langle x\rangle^{\alpha_{1}} f \partial_{x} g\right\|_{L_{T}^{1}\left(L_{x}^{2}\right)+L_{T}^{4 / 3}\left(L_{x}^{1}\right)}$. We write

$$
\begin{aligned}
D_{x}^{s_{1}}\left(\langle x\rangle^{\alpha_{1}} f \partial_{x} g\right)= & \langle x\rangle^{\alpha_{1}} f D_{x}^{s_{1}} \partial_{x} g+\left(D_{x}^{s_{1}}\langle x\rangle^{\alpha_{1}} f\right) \partial_{x} g \\
& +\left(D_{x}^{s_{1}}\left(\langle x\rangle^{\alpha_{1}} f \partial_{x} g\right)-\langle x\rangle^{\alpha_{1}} f D_{x}^{s_{1}} \partial_{x} g-\left(D_{x}^{s_{1}}\langle x\rangle^{\alpha_{1}} f\right) \partial_{x}\right) .
\end{aligned}
$$

Then, $L_{T}^{2}\left(L_{x}^{2}\right) \subset L_{T}^{1}\left(L_{x}^{2}\right)$ and Lemma 3.4 yield

$$
\begin{aligned}
& \left\|D_{x}^{s_{1}}\langle x\rangle^{\alpha_{1}} f \partial_{x} g\right\|_{L_{T}^{1}\left(L_{x}^{2}\right)+L_{T}^{4 / 3}\left(L_{x}^{1}\right)} \\
& \leq C T^{1 / 2}\left\|\langle x\rangle^{\alpha_{1}} f\right\|_{L_{x}^{\tilde{p}_{1}}\left(L_{T}^{\tilde{r}_{1}}\right)}\left\|\left\langle D_{x}\right\rangle^{s_{1}} \partial_{x} g\right\|_{L_{x}^{\tilde{p}_{2}}\left(L_{T}^{\tilde{r}_{2}}\right)}+\left\|\left(D_{x}^{s_{1}}\langle x\rangle^{\alpha_{1}} f\right) \partial_{x} g\right\|_{L_{T}^{4 / 3}\left(L_{x}^{1}\right)} \\
& \leq C T^{1 / 2}\left\|\langle x\rangle^{\alpha_{1}} f\right\|_{L_{x}^{\tilde{p}_{1}}\left(L_{T}^{\tilde{r}_{1}}\right)}\left\|\left\langle D_{x}\right\rangle^{s_{1}+1} g\right\|_{L_{x}^{\tilde{p}_{2}}\left(L_{T}^{\tilde{r}_{2}}\right)}+C T^{3 / 4}\|f\|_{\text {initial }}\|g\|_{\text {initial }},
\end{aligned}
$$


where

$$
\begin{aligned}
& 1 / \tilde{p}_{1}+1 / \tilde{p}_{2}=1 / 2, \quad 1 / \tilde{r}_{1}+1 / \tilde{r}_{2}=1 / 2 \\
& 1 / \tilde{p}_{2}=(1-\tilde{\theta}) / 2+\tilde{\theta} \varepsilon, \quad 1 / \tilde{r}_{2}=(1-\tilde{\theta}) / \infty+\tilde{\theta} / 2,
\end{aligned}
$$

with $s_{1}+1=(1-\tilde{\theta}) \tilde{\mu} / 2+\tilde{\theta}\left(s_{1}+1+\tilde{\mu} / 2\right)$ for small $\tilde{\mu} \in(0, \mu)$. By the interpolation (Corollary 3.6), we have

$$
\left\|\left\langle D_{x}\right\rangle^{s_{1}+1} g\right\|_{L_{x}^{\tilde{p}_{2}\left(L_{T}^{\tilde{r}_{2}}\right)}} \leq C\left\|\left\langle D_{x}\right\rangle^{\tilde{\mu}}\langle x\rangle^{\tilde{\rho}} g\right\|_{L_{x}^{2}\left(L_{T}^{\infty}\right)}^{1-\tilde{\tilde{C}}}\left\|\left\langle D_{x}\right\rangle^{s_{1}+1+\tilde{\mu}}\langle x\rangle^{-\rho} g\right\|_{L_{x}^{1 / \varepsilon}\left(L_{T}^{2}\right)}^{\tilde{\tilde{\theta}}},
$$

where $\tilde{\rho}$ satisfies $0=(1-\tilde{\theta}) \tilde{\rho}+\tilde{\theta}(-\rho)$. Since the smallness of $\rho$ and $\tilde{\mu}$ allows $\tilde{\rho}<\alpha_{1}$ and $s_{1}+1+\tilde{\mu}<s+1 / 2$, we see that

$$
\begin{aligned}
\left\|\left\langle D_{x}\right\rangle^{s_{1}+1} g\right\|_{L_{x}^{\tilde{p}_{2}}\left(L_{T}^{\tilde{r}_{2}}\right)} & \leq C\|g\|_{\text {maxim }}^{1-\tilde{\theta}}\left(\|g\|_{\text {smooth }}+T^{1 / 2}\|g\|_{\text {initial }}\right)^{\tilde{\theta}} \\
& \leq C\|g\|_{Y_{T} .}
\end{aligned}
$$

As for $\left\|\langle x\rangle^{\alpha_{1}} f\right\|_{L_{x}^{\tilde{p}_{1}}\left(L_{T}^{\tilde{r}_{1}}\right)}$, we remark that $1 / 2-1 / \tilde{p}_{1}=1 / \tilde{p}_{2}=\tilde{\mu} / 4\left(s_{1}+1\right)+$ $\varepsilon\left(s_{1}+1-\tilde{\mu} / 2\right) /\left(s_{1}+1\right)<\mu$ for small $\tilde{\mu}$ and $\varepsilon$. Therefore, the Hardy-LittlewoodSobolev inequality gives

$$
\begin{aligned}
\left\|\langle x\rangle^{\alpha_{1}} f\right\|_{L_{x}^{\tilde{p}_{1}\left(L_{T}^{\tilde{r}_{1}}\right)}} & \leq C\left\|\left\langle D_{x}\right\rangle^{1 / \tilde{p}_{2}}\langle x\rangle^{\alpha_{1}} f\right\|_{L_{x}^{2}\left(L_{T}^{\tilde{r}_{1}}\right)} \\
& \leq C T^{1 / \tilde{r}_{1}}\|f\|_{\text {maxim }} \\
& \leq C\|f\|_{Y_{T}}
\end{aligned}
$$

Combining (5.8)-(5.10), we obtain (5.2).

The lemma given below is concerning the estimates of the remainder term.

Lemma 5.2. Let $s^{\prime}$ slightly less than $s$. Then, there exist $C_{\varphi}>0$ and small positive constant $\beta$ such that

$$
\begin{aligned}
& \left\|R_{\nu}\left(\varphi, u_{\nu}\right)\right\|_{L_{T}^{1}\left(H_{x}^{s, 0}\right)} \leq C_{\varphi} T\left\|u_{\nu}\right\|_{Y_{T}} \\
& \left\|R_{\nu}\left(\varphi, u_{\nu}\right)-R_{\nu^{\prime}}\left(\varphi, u_{\nu^{\prime}}\right)\right\|_{L_{T}^{1}\left(H_{x}^{s^{\prime}, 0}\right)} \\
& \quad \leq C_{\varphi} T\left\|u_{\nu}-u_{\nu^{\prime}}\right\|_{L_{T}^{\infty}\left(H_{x}^{s^{\prime}, 0}\right)}+C_{\varphi} T\left(\nu^{\beta}+\nu^{\prime \beta}\right)\left(\left\|u_{\nu}\right\|_{Y_{T}}+\left\|u_{\nu^{\prime}}\right\|_{Y_{T}}\right) .
\end{aligned}
$$

Proof of Lemma 5.2. Since $K_{\nu} \varphi \eta_{\nu} * \partial_{x}+\left[K_{\nu},\left(1-\psi\left(i^{-1} \partial_{x}\right)\right) \mathcal{H}_{x} \partial_{x}^{2}\right] \in S^{0}$, this is $H_{x}^{s, 0}$-bounded and its operator norm is estimated in terms of large order derivatives of $\varphi$. Also, $\left[\psi\left(i^{-1} \partial_{x}\right) \mathcal{H}_{x} \partial_{x}^{2}, K_{\nu}\right] \in \mathcal{B}\left(H_{x}^{s, 0}\right)$ since $\psi\left(i^{-1} \partial_{x}\right) \mathcal{H}_{x} \partial_{x}^{2} \in$ $\mathcal{B}\left(H_{x}^{s, 0}\right)$. Hence, we obtain (5.11). The estimate (5.12) likewise follows. We note that $\nu^{\beta}$ and $\nu^{\prime \beta}$ appear in the estimates of $K_{\nu}-K_{\nu^{\prime}}$ and $\eta_{\nu}-\eta_{\nu^{\prime}}$ (The slight loss of regularity occurs in these estimates). 


\section{$\S 6 . \quad$ Proof of Theorem 1.1}

The local existence of the modified solution $u_{\nu}$ to (1.2) follows from Lemmas 3.1, 4.1, 4.2 and the strong smoothing property of $\eta_{\nu} *$. Furthermore, this local solution is continuated as long as $\left\|u_{\nu}(t)\right\|_{X^{s}}<\infty$. We note that $\left\|u_{\nu}\right\|_{Y_{T}}$ is continuous with respective to $T$. Let $\left\|u_{0}\right\|_{X^{s}} \leq \delta_{0}$ and $T_{\nu}=\sup \left\{T^{\prime} ;\left\|u_{\nu}\right\|_{Y_{\tau}}<\right.$ $2 \tilde{C}_{\varphi} \delta_{0}$ for $\left.0<\tau<T^{\prime}\right\}$ (The large positive constant $\tilde{C}_{\varphi}$ may diverge as $\varphi \rightarrow u_{0}$ in $X^{s}$, and it will be specified later). The modified solutions $u_{\nu}$ and $v_{\nu}=K_{\nu} u_{\nu}$ respectively satisfy

$$
\begin{aligned}
& u_{\nu}=V(t) u_{0}-G(t)\left(u_{\nu} \eta_{\nu} * \partial_{x} u_{\nu}\right), \\
& v_{\nu}=V(t) K_{\nu} u_{0}-G(t) K_{\nu}\left(\left(u_{\nu}-\varphi\right) \eta_{\nu} * \partial_{x} u_{\nu}\right)-G(t) R_{\nu}\left(\varphi, u_{\nu}\right) .
\end{aligned}
$$

The uniform lower bound of $T_{\nu}$ and convergence of $u_{\nu}$ as $\nu \downarrow 0$ are obtained by the following proposition.

Proposition 6.1. The following assertions hold.

1. There exists some $T_{0}>0$ such that $\inf _{\nu>0} T_{\nu} \geq T_{0}$.

2. For some $T \in\left(0, T_{0}\right)$, we have

$$
\begin{aligned}
\left\|u_{\nu}\right\|_{Y_{T}} & \leq 2 C_{0} \delta_{0}, \\
\left\|u_{\nu}-u_{\nu^{\prime}}\right\|_{Y_{T}^{\prime}} & \leq C_{\varphi}\left(\nu^{\beta}+\nu^{\prime \beta}\right),
\end{aligned}
$$

where $\|\cdot\|_{Y_{T}^{\prime}}$ is given by

$$
\begin{gathered}
\|f\|_{Y_{T}^{\prime}}=\|f\|_{L_{T}^{\infty}\left(H_{x}^{s^{\prime}, 0} \cap H_{x}^{s_{1}^{\prime}, \alpha_{1}}\right)}+\left\|\langle x\rangle^{-\rho}\left\langle D_{x}\right\rangle^{s^{\prime}+1 / 2} f\right\|_{L_{x}^{1 / \varepsilon}\left(L_{T}^{2}\right)} \\
+\left\|\left\langle D_{x}\right\rangle^{\mu^{\prime}}\langle x\rangle^{\alpha_{1}} f\right\|_{L_{x}^{2}\left(L_{T}^{\infty}\right)}
\end{gathered}
$$

with $s^{\prime}\left(\right.$ resp. $\left.s_{1}^{\prime}, \mu^{\prime}\right)$ slightly less than $s\left(\right.$ resp. $\left.s_{1}, \mu\right)$.

To prove Proposition 6.1, we need two lemmas. The first lemma suggests that the estimates of $v_{\nu}$ gives those of $u_{\nu}$.

Lemma 6.2. Let $s^{\prime}<s$ and $T \in\left(0, T_{\nu}\right)$. Then, there exist positive constants $C, C_{\varphi}$ and $\beta$ such that

$$
\begin{aligned}
& \left\|u_{\nu}\right\|_{L_{T}^{\infty}\left(H_{x}^{s, 0}\right)} \\
& \quad \leq C\left\|v_{\nu}\right\|_{L_{T}^{\infty}\left(H_{x}^{s, 0}\right)}+C_{\varphi}\left\|u_{\nu}\right\|_{L_{T}^{\infty}\left(L_{x}^{2}\right)}, \\
& \quad\left\|\langle x\rangle^{-\rho}\left\langle D_{x}\right\rangle^{s+1 / 2} u_{\nu}\right\|_{L_{x}^{1 / \varepsilon}\left(L_{T}^{2}\right)} \\
& \quad \leq C\left\|\langle x\rangle^{-\rho}\left\langle D_{x}\right\rangle^{s+1 / 2} v_{\nu}\right\|_{L_{x}^{1 / \varepsilon}\left(L_{T}^{2}\right)}+C_{\varphi} T^{\beta}\left\|u_{\nu}\right\|_{L_{T}^{\infty}\left(H_{x}^{s, 0}\right)},
\end{aligned}
$$


(6.7)

$$
\begin{aligned}
(6.7) \| u_{\nu}- & u_{\nu^{\prime}} \|_{L_{T}^{\infty}\left(H_{x}^{s^{\prime}, 0}\right)} \\
\leq & C\left\|v_{\nu}-v_{\nu^{\prime}}\right\|_{L_{T}^{\infty}\left(H_{x}^{s^{\prime}, 0}\right)}+C_{\varphi}\left\|u_{\nu}-u_{\nu^{\prime}}\right\|_{L_{T}^{\infty}\left(L_{x}^{2}\right)} \\
& \quad+C_{\varphi}\left(\nu^{\beta}+\nu^{\prime \beta}\right)\left(\left\|u_{\nu}\right\|_{Y_{T}}+\left\|u_{\nu^{\prime}}\right\|_{Y_{T}}\right), \\
(6.8)\left\|\langle x\rangle^{-\rho}\left\langle D_{x}\right\rangle^{s^{\prime}+1 / 2}\left(u_{\nu}-u_{\nu^{\prime}}\right)\right\|_{L_{x}^{1 / \varepsilon}\left(L_{T}^{2}\right)} & \\
\leq & C\left\|\langle x\rangle^{-\rho}\left\langle D_{x}\right\rangle^{s^{\prime}+1 / 2}\left(v_{\nu}-v_{\nu^{\prime}}\right)\right\|_{L_{x}^{1 / \varepsilon}\left(L_{T}^{2}\right)} \\
& +C_{\varphi} T^{\beta}\left\|u_{\nu}-u_{\nu^{\prime}}\right\|_{L_{T}^{\infty}\left(H_{x}^{s^{\prime}, 0}\right)}+C_{\varphi}\left(\nu^{\beta}+\nu^{\prime \beta}\right)\left(\left\|u_{\nu}\right\|_{Y_{T}}+\left\|u_{\nu^{\prime}}\right\|_{Y_{T}}\right),
\end{aligned}
$$

where $C_{\varphi}$ is allowed to diverge as $\varphi \rightarrow u_{0}$ in $X^{s}$.

Proof of Lemma 6.2. Since $\partial_{x} K_{\nu}=K_{\nu} \partial_{x}-(\pi / 2)^{1 / 2} \varphi K_{\nu} \mathcal{H}_{x}(1-$ $\left.\psi\left(i^{-1} \partial_{x}\right)\right) \eta_{\nu} *$, we see that

$$
\left\langle D_{x}\right\rangle^{s-1} \partial_{x} v_{\nu}=K_{\nu}\left\langle D_{x}\right\rangle_{x}^{s-1} \partial_{x} u_{\nu}+r\left(u_{\nu}\right)
$$

where $r\left(u_{\nu}\right)$ is a remainder with $\left\|r\left(u_{\nu}\right)\right\|_{L_{T}^{\infty}\left(L_{x}^{2}\right)}$ bounded by $C_{\varphi}\left\|u_{\nu}\right\|_{L_{T}^{\infty}\left(L_{x}^{2}\right)}+$ $\kappa\left\|u_{\nu}\right\|_{L_{T}^{\infty}\left(H_{x}^{s, 0}\right)}$ with $\kappa>0$ small. Let $\widetilde{K}_{\nu}(\varphi)\left(=\widetilde{K}_{\nu}\right)$ be the pseudo-differential operator of the symbol:

$$
\widetilde{K}_{\nu}(x, \xi)=\exp \left(-\sqrt{\frac{\pi}{2}} \frac{i \xi}{|\xi|}(1-\psi(\xi)) \hat{\eta}(\nu \xi) \int_{-\infty}^{x} \varphi(y) d y\right)
$$

We here note that $\widetilde{K}_{\nu}$ plays a role like an inverse of $K_{\nu}$ and, precisely speaking, $I-\widetilde{K}_{\nu} K_{\nu} \in S^{-1}$ uniformly in $\nu \in(0,1]$. Applying $\widetilde{K}_{\nu}$ to $(6.9)$, we have

$$
\left\langle D_{x}\right\rangle^{s-1} \partial_{x} u_{\nu}=\widetilde{K}_{\nu}\left\langle D_{x}\right\rangle_{x}^{s-1} \partial_{x} v_{\nu}+\left(I-\widetilde{K}_{\nu} K_{\nu}\right)\left\langle D_{x}\right\rangle_{x}^{s-1} \partial_{x} u_{\nu}-\widetilde{K}_{\nu} r\left(u_{\nu}\right) .
$$

Thus, it follows from Lemma 3.7 that

$$
\begin{aligned}
\left\|\left\langle D_{x}\right\rangle^{s-1} \partial_{x} u_{\nu}\right\|_{L_{T}^{\infty}\left(L_{x}^{2}\right) \leq} & \left.C\left\|v_{\nu}\right\|_{L_{T}^{\infty}\left(H_{x}^{s, 0}\right)}+C_{\varphi}\left\|u_{\nu}\right\|_{L_{T}^{\infty}\left(H_{x}^{s-1,0}\right)}\right) \\
& +\left(C_{\varphi}\left\|u_{\nu}\right\|_{L_{T}^{\infty}\left(L_{x}^{2}\right)}+\kappa\left\|u_{\nu}\right\|_{L_{T}^{\infty}\left(H_{x}^{s, 0}\right)}\right) .
\end{aligned}
$$

Using $\left\|u_{\nu}\right\|_{L_{T}^{\infty}\left(H_{x}^{s-1,0}\right)} \leq C\left\|u_{\nu}\right\|_{L_{T}^{\infty}\left(L_{x}^{2}\right)}+\kappa\left\|u_{\nu}\right\|_{L_{T}^{\infty}\left(H_{x}^{s, 0}\right)}$ and taking $\kappa$ sufficiently small, we obtain (6.5). We next prove (6.6). Write

$$
\begin{aligned}
\left\langle D_{x}\right\rangle^{s+1 / 2} u_{\nu}= & \widetilde{K}_{\nu}\left\langle D_{x}\right\rangle^{s+1 / 2} v_{\nu}+\left(I-\widetilde{K}_{\nu} K_{\nu}\right)\left\langle D_{x}\right\rangle^{s+1 / 2} u_{\nu} \\
& -\widetilde{K}_{\nu}\left[\left\langle D_{x}\right\rangle^{s+1 / 2}, K_{\nu}\right] u_{\nu} .
\end{aligned}
$$


Then, Lemma 3.7, $L_{T}^{2}\left(L_{x}^{1 / \varepsilon}\right) \subset L_{x}^{1 / \varepsilon}\left(L_{T}^{2}\right)$ and $H_{x}^{1 / 2,0} \subset L_{x}^{1 / \varepsilon}$ yield

$$
\begin{aligned}
\left\|\langle x\rangle^{-\rho}\left\langle D_{x}\right\rangle^{s+1 / 2} u_{\nu}\right\|_{L_{x}^{1 / \varepsilon}\left(L_{T}^{2}\right)} \leq & \left\|\widetilde{K}_{\nu}\langle x\rangle^{-\rho}\left\langle D_{x}\right\rangle^{s+1 / 2} v_{\nu}\right\|_{L_{x}^{1 / \varepsilon}\left(L_{T}^{2}\right)} \\
& +\left\|\left[\langle x\rangle^{-\rho}, \widetilde{K}_{\nu}\right]\left\langle D_{x}\right\rangle^{s+1 / 2} v_{\nu}\right\|_{L_{T}^{2}\left(L_{x}^{1 / \varepsilon}\right)} \\
& +\left\|\langle x\rangle^{-\rho}\left(I-\widetilde{K}_{\nu} K_{\nu}\right)\left\langle D_{x}\right\rangle^{s+1 / 2} u_{\nu}\right\|_{L_{T}^{2}\left(L_{x}^{1 / \varepsilon}\right)} \\
& +\left\|\langle x\rangle^{-\rho} \widetilde{K}_{\nu}\left[\left\langle D_{x}\right\rangle^{s+1 / 2}, K_{\nu}\right] u_{\nu}\right\|_{L_{T}^{2}\left(L_{x}^{1 / \varepsilon}\right)} \\
\leq & C\left\|\langle x\rangle^{-\rho}\left\langle D_{x}\right\rangle^{s+1 / 2} v_{\nu}\right\|_{L_{x}^{1 / \varepsilon}\left(L_{T}^{2}\right)} \\
& +C T^{1 / 2}\left\|\left[\langle x\rangle^{-\rho}, \widetilde{K}_{\nu}\right]\left\langle D_{x}\right\rangle^{s+1 / 2} v_{\nu}\right\|_{L_{T}^{\infty}\left(H_{x}^{1 / 2,0}\right)} \\
& +C T^{1 / 2}\left\|\left(I-\widetilde{K}_{\nu} K_{\nu}\right)\left\langle D_{x}\right\rangle^{s+1 / 2} u_{\nu}\right\|_{L_{T}^{\infty}\left(H_{x}^{1 / 2,0}\right)} \\
& +C T^{1 / 2}\left\|\widetilde{K}_{\nu}\left[\left\langle D_{x}\right\rangle^{s+1 / 2}, K_{\nu}\right] u_{\nu}\right\|_{L_{T}^{\infty}\left(H_{x}^{1 / 2,0}\right)}
\end{aligned}
$$

Since $\sigma\left(\left[\langle x\rangle^{-\rho}, \widetilde{K}_{\nu}\right]\right) \in S^{-1}$ and $\sigma\left(\left[\left\langle D_{x}\right\rangle^{s+1 / 2}, K_{\nu}\right]\right) \in S^{s-1 / 2}$, we see that

$$
\begin{aligned}
\left\|\langle x\rangle^{-\rho}\left\langle D_{x}\right\rangle^{s+1 / 2} u_{\nu}\right\|_{L_{x}^{1 / \varepsilon}\left(L_{T}^{2}\right)} \leq & C\left\|\langle x\rangle^{-\rho}\left\langle D_{x}\right\rangle^{s+1 / 2} v_{\nu}\right\|_{L_{x}^{1 / \varepsilon}\left(L_{T}^{2}\right)} \\
& +C_{\varphi} T^{1 / 2}\left(\left\|v_{\nu}\right\|_{L_{T}^{\infty}\left(H_{x}^{s, 0}\right)}+\left\|u_{\nu}\right\|_{L_{T}^{\infty}\left(H_{x}^{s, 0}\right)}\right) .
\end{aligned}
$$

Since $\left\|v_{\nu}\right\|_{L_{T}^{\infty}\left(H_{x}^{s, 0}\right)} \leq C_{\varphi}\left\|u_{\nu}\right\|_{L_{T}^{\infty}\left(H_{x}^{s, 0}\right)}$, we obtain (6.6). The estimates (6.7) and (6.8) follow in the similar way. We note that, to derive $\nu^{\beta}+\nu^{\prime \beta}$ in the estimate of $K_{\nu}-K_{\nu^{\prime}}$, the slight loss of regularity occurs.

The second lemma allows to make the nonlinearity in (6.2) small enough by taking $\varphi$ close to $u_{0}$ and $T>0$ small.

Lemma 6.3. $\quad$ There exist positive constants $C, C_{\varphi}$ and $\beta$ such that

$$
\left\|u_{\nu}-\varphi\right\|_{\text {maxim }} \leq C\left\|u_{0}-\varphi\right\|_{X^{s}}+C_{\varphi} T^{\beta}\left(1+\left\|u_{\nu}\right\|_{Y_{T}}\right)^{2},
$$

where $C_{\varphi}$ may diverge as $\varphi \rightarrow u_{0}$ in $X^{s}$. Furthermore, taking $\varphi$ close to $u_{0}$ in $X^{s}$, we have

$$
\left\|u_{\nu}\right\|_{s m o o t h} \leq C\left\|u_{0}-\varphi\right\|_{X^{s}}+C_{\varphi} T^{\beta}\left(1+\left\|u_{\nu}\right\|_{Y_{T}}\right)^{3} .
$$

Proof of Lemma 6.3. We first note that $\left\|\eta_{\nu} * u_{\nu}\right\|_{Y_{T}} \leq C\left\|u_{\nu}\right\|_{Y_{T}}$. In fact, by regarding $\eta_{\nu^{*}}=\sqrt{2 \pi} \hat{\eta}\left(\nu i^{-1} \partial_{x}\right)$, the symbol of $\left[\langle x\rangle^{\alpha}, \hat{\eta}\left(\nu i^{-1} \partial_{x}\right)\right]$ belongs to $S^{-1}$ uniformly in $\nu \in(0,1]$ if $\alpha \leq 1$, which yields $\left[\langle x\rangle^{\alpha}, \hat{\eta}\left(\nu i^{-1} \partial_{x}\right)\right] \in$ $\mathcal{B}\left(L_{T}^{r}\left(L_{x}^{p}\right)\right) \cap \mathcal{B}\left(L_{x}^{p}\left(L_{T}^{r}\right)\right)$ with the operator norms independent of $\nu$. Then, it is 
easy to see that

$$
\begin{gathered}
\left\|\eta_{\nu} * u_{\nu}\right\|_{\text {initial }} \leq C\left\|u_{\nu}\right\|_{\text {initial }}, \\
\left\|\eta_{\nu} * u_{\nu}\right\|_{\text {maxim }} \leq C\left\|u_{\nu}\right\|_{\text {maxim }} .
\end{gathered}
$$

The estimate of $\left\|\eta_{\nu} * u_{\nu}\right\|_{\text {smooth }}$ follows from

$$
\begin{aligned}
\left\|\eta_{\nu} * u_{\nu}\right\|_{\text {smooth }} \leq & \left\|\langle x\rangle^{-\rho} \hat{\eta}\left(\nu i^{-1} \partial_{x}\right)\langle x\rangle^{\rho} \cdot\langle x\rangle^{-\rho}\left\langle D_{x}\right\rangle^{s+1 / 2} u_{\nu}\right\|_{L_{x}^{1 / \varepsilon}\left(L_{T}^{2}\right)} \\
\leq & \left\|\hat{\eta}\left(\nu i^{-1} \partial_{x}\right) \cdot\langle x\rangle^{-\rho}\left\langle D_{x}\right\rangle^{s+1 / 2} u_{\nu}\right\|_{L_{x}^{1 / \varepsilon}\left(L_{T}^{2}\right)} \\
& +\left\|\langle x\rangle^{-\rho}\left[\hat{\eta}\left(\nu i^{-1} \partial_{x}\right),\langle x\rangle^{\rho}\right]\langle x\rangle^{-\rho}\left\langle D_{x}\right\rangle^{s+1 / 2} u_{\nu}\right\|_{L_{x}^{1 / \varepsilon}\left(L_{T}^{2}\right)} \\
\leq & C\left\|u_{\nu}\right\|_{\text {smooth. }} .
\end{aligned}
$$

Therefore, we have $\left\|\eta_{\nu} * u_{\nu}\right\|_{Y_{T}} \leq C\left\|u_{\nu}\right\|_{Y_{T}}$.

Applying Lemmas 4.2 and 5.1 to (6.1), we have

$$
\begin{aligned}
\left\|u_{\nu}-\varphi\right\|_{\text {maxim }} \leq & \left\|V(t) u_{0}-\varphi\right\|_{\text {maxim }}+C T^{1 / 2}\left\|D_{x}^{s-1 / 2}\left(u_{\nu} \eta_{\nu} * \partial_{x} u_{\nu}\right)\right\|_{L_{x}^{1}\left(L_{T}^{2}\right)} \\
& +C\left\|\left\langle D_{x}\right\rangle^{s_{1}}\langle x\rangle^{\alpha_{1}}\left(u_{\nu} \eta_{\nu} * \partial_{x} u_{\nu}\right)\right\|_{L_{T}^{1}\left(L_{x}^{2}\right)+L_{T}^{4 / 3}\left(L_{x}^{1}\right)} \\
\leq & \left\|V(t) u_{0}-\varphi\right\|_{\text {maxim }}+C T^{\beta}\left\|u_{\nu}\right\|_{Y_{T}}^{2} .
\end{aligned}
$$

In virtue of Lemma 4.2, the first term in (6.12) is estimated as

$$
\begin{aligned}
\left\|V(t) u_{0}-\varphi\right\|_{\text {maxim }} & \leq\left\|V(t)\left(u_{0}-\varphi\right)\right\|_{\text {maxim }}+\|V(t) \varphi-\varphi\|_{\text {maxim }} \\
& \leq C\left\|u_{0}-\varphi\right\|_{X^{s}}+C T\|\varphi\|_{H_{x}^{m, n}}
\end{aligned}
$$

with $m, n>0$ large. Thus, combining (6.12) and (6.13), we obtain (6.10). We next prove (6.11). Applying Hölder's inequality and Lemma 3.1 to (6.2), we have

$$
\begin{aligned}
\left\|v_{\nu}\right\|_{\text {smooth }} \leq & C\left\|\left\langle D_{x}\right\rangle^{s+1 / 2} V(t) K_{\nu} u_{0}\right\|_{L_{x}^{\infty}\left(L_{T}^{2}\right)} \\
& +C\left\|\left\langle D_{x}\right\rangle^{s-1 / 2} K_{\nu}\left(\left(u_{\nu}-\varphi\right) \eta_{\nu} * \partial_{x} u_{\nu}\right)\right\|_{L_{x}^{1}\left(L_{T}^{2}\right)} \\
& +C\left\|R_{\nu}\left(\varphi, u_{\nu}\right)\right\|_{L_{T}^{1}\left(H_{x}^{s, 0}\right)}
\end{aligned}
$$

Note that, to obtain the estimate of $R_{\nu}\left(\varphi, u_{\nu}\right)$ in the above inequality, we used

$$
\begin{aligned}
& \left\|\left\langle D_{x}\right\rangle^{s+1 / 2} G R_{\nu}\left(\varphi, u_{\nu}\right)\right\|_{L_{x}^{\infty}\left(L_{T}^{2}\right)} \\
& \quad \leq \int_{0}^{T}\left\|\left\langle D_{x}\right\rangle^{s+1 / 2} V(t) V(-\tau) R_{\nu}\left(\varphi, u_{\nu}\right)\right\|_{L_{x}^{\infty}\left(L_{T}^{2}\right)} d \tau \\
& \quad \leq C \int_{0}^{T}\left\|\left\langle D_{x}\right\rangle^{s} V(-\tau) R_{\nu}\left(\varphi, u_{\nu}\right)\right\|_{L_{x}^{2}} d \tau .
\end{aligned}
$$


Lemmas 3.1, 3.2 and $L_{T}^{2}\left(L_{x}^{\infty}\right) \subset L_{x}^{\infty}\left(L_{T}^{2}\right)$ give

(The first term in (6.14))

$$
\begin{aligned}
\leq & \left\|\left\langle D_{x}\right\rangle^{1 / 2} V(t) K_{\nu}\left\langle D_{x}\right\rangle^{s}\left(u_{0}-\varphi\right)\right\|_{L_{x}^{\infty}\left(L_{T}^{2}\right)} \\
& +T^{1 / 4}\left\|\left\langle D_{x}\right\rangle^{1 / 2} V(t) K_{\nu}\left\langle D_{x}\right\rangle^{s} \varphi\right\|_{L_{T}^{4}\left(L_{x}^{\infty}\right)} \\
& +T^{1 / 4}\left\|V(t)\left\langle D_{x}\right\rangle^{1 / 2}\left[\left\langle D_{x}\right\rangle^{s}, K_{\nu}\right] u_{0}\right\|_{L_{T}^{4}\left(L_{x}^{\infty}\right)} \\
\leq & C\left\|u_{0}-\varphi\right\|_{H_{x}^{s, 0}}+C_{\varphi} T^{1 / 4}+C_{\varphi} T^{1 / 4}\left\|u_{0}\right\|_{H_{x}^{s-1 / 2,0}} \\
\leq & C\left\|u_{0}-\varphi\right\|_{H_{x}^{s, 0}}+C_{\varphi} T^{1 / 4} .
\end{aligned}
$$

Applying Lemmas 5.1, 6.3 (6.10) and

$$
\begin{aligned}
\left\|\eta_{\nu} * u_{\nu}\right\|_{\text {maxim }} & \leq C\left\|u_{\nu}\right\|_{\text {maxim }}, \\
\left\|u_{\nu}\right\|_{\text {maxim }} & \leq\left\|u_{\nu}-\varphi\right\|_{\text {maxim }}+C\|\varphi\|_{H_{x}^{s_{1}, \alpha_{1}}}, \\
\left\|\eta_{\nu} * u_{\nu}\right\|_{\text {smooth }} & \leq\left\|u_{\nu}\right\|_{\text {smooth }}+C T^{1 / 2}\left\|u_{\nu}\right\|_{\text {initial }},
\end{aligned}
$$

we have

(6.16) (The second term in (6.14))

$$
\begin{aligned}
\leq & C\left\|u_{\nu}-\varphi\right\|_{\text {maxim }}\left\|u_{\nu}\right\|_{\text {smooth }} \\
& +C\left\|u_{\nu}-\varphi\right\|_{\text {maxim }}^{\theta}\left\|u_{\nu}-\varphi\right\|_{\text {smooth }}^{1-\theta}\left\|u_{\nu}\right\|_{\text {maxim }}^{1-\theta}\left\|u_{\nu}\right\|_{\text {smooth }}^{\theta} \\
& +C_{\varphi} T^{\beta}\left(1+\left\|u_{\nu}\right\| \|_{Y_{T}}\right)^{2} \\
\leq & C\left(\left\|u_{0}-\varphi\right\|_{X^{s}}+\left\|u_{0}-\varphi\right\|_{X^{s}}^{\theta}\right)\left\|u_{\nu}\right\|_{\text {smooth }} \\
& +C_{\varphi} T^{\beta}\left(1+\left\|u_{\nu}\right\|_{Y_{T}}\right)^{3} .
\end{aligned}
$$

Also, Lemma 5.2 gives

$$
\text { (The third term in }(6.14)) \leq C_{\varphi} T\left\|u_{\nu}\right\|_{Y_{T}} .
$$

Combining (6.14)-(6.17) and applying Lemma 6.2, we obtain (6.11).

Proof of Proposition 6.1. Applying Lemmas 4.1, 4.2 and the nonlinear estimates as in Lemma 5.1 to (6.1), we have

$$
\begin{aligned}
\left.\left\|u_{\nu}\right\|_{L_{T}^{\infty}\left(H_{x}^{s_{1}}, \alpha_{1}\right.}\right) & +\left\|u_{\nu}\right\|_{\text {maxim }} \\
\leq & C\left\|u_{0}\right\|_{X^{s}}+C T^{1 / 2}\left\|D_{x}^{s-1 / 2}\left(u_{\nu} \eta_{\nu} * \partial_{x} u_{\nu}\right)\right\|_{L_{x}^{1}\left(L_{T}^{2}\right)} \\
& +C\left\|\left\langle D_{x}\right\rangle^{s_{1}}\left(\langle x\rangle^{\alpha_{1}} u_{\nu} \eta_{\nu} * \partial_{x} u_{\nu}\right)\right\|_{L_{T}^{1}\left(L_{x}^{2}\right)+L_{T}^{4 / 3}\left(L_{x}^{1}\right)} \\
\leq & C\left\|u_{0}\right\|_{X^{s}}+C T^{\beta}\left\|u_{\nu}\right\|_{Y_{T}}^{2} .
\end{aligned}
$$


We next apply Lemmas 3.1 and 5.2 to (6.2). Then, it follows from Lemmas 5.1 and 5.2 that

$$
\begin{aligned}
\left\|v_{\nu}\right\|_{L_{T}^{\infty}\left(H_{x}^{s, 0}\right)}+\left\|v_{\nu}\right\|_{\text {smooth }} & \\
\leq & \left\|v_{\nu}\right\|_{L_{T}^{\infty}\left(H_{x}^{s, 0}\right)}+C\left\|\left\langle D_{x}\right\rangle^{s+1 / 2} v_{\nu}\right\|_{L_{x}^{\infty}\left(L_{T}^{2}\right)} \\
\leq & C\left\|u_{0}\right\|_{X^{s}}+C\left\|K_{\nu}\left(\left(u_{\nu}-\varphi\right) \eta_{\nu} * \partial_{x} u_{\nu}\right)\right\|_{L_{x}^{1}\left(L_{T}^{2}\right)} \\
& +C\left\|R_{\nu}\left(\varphi, u_{\nu}\right)\right\|_{L_{T}^{1}\left(H_{x}^{s, 0}\right)} \\
\leq & C\left\|u_{0}\right\|_{X^{s}}+C\left\|u_{\nu}-\varphi\right\|_{\text {maxim }}\left\|u_{\nu}\right\|_{\text {smooth }} \\
& +C\left\|u_{\nu}-\varphi\right\|_{\text {maxim }}^{\theta}\|\varphi\|_{H_{x}^{s_{1}, \alpha_{1}}}^{1-\theta}\left\|u_{\nu}\right\|_{\text {smooth }}+C_{\varphi} T^{\beta}\left(1+\left\|u_{\nu}\right\|_{Y_{T}}\right)^{2} .
\end{aligned}
$$

Thus, by Lemmas 6.2 and $6.3(6.10)$,

$$
\begin{aligned}
\left\|u_{\nu}\right\|_{L_{T}^{\infty} H_{x}^{s, 0}}+\left\|u_{\nu}\right\|_{\text {smooth }} & \\
\leq & C_{\varphi}\left\|u_{0}\right\|_{X^{s}}+C\left(\left\|u_{\nu}-\varphi\right\|_{X^{s}}+\left\|u_{\nu}-\varphi\right\|_{X^{s}}^{\theta}\right)\left\|u_{\nu}\right\|_{Y_{T}} \\
& +C_{\varphi} T^{\beta}\left(1+\left\|u_{\nu}\right\|_{Y_{T}}\right)^{3} .
\end{aligned}
$$

Combining (6.18) and (6.19), we have

$$
\begin{aligned}
\left\|u_{\nu}\right\|_{Y_{T}} \leq & \tilde{C}_{\varphi} \delta_{0}+C\left(\left\|u_{0}-\varphi\right\|_{X^{s}}+\left\|u_{0}-\varphi\right\|_{X^{s}}^{\theta}\right) \cdot 2 \tilde{C}_{\varphi} \delta_{0} \\
& +C_{\varphi} T^{\beta}\left(1+2 \tilde{C}_{\varphi} \delta_{0}\right)^{3} .
\end{aligned}
$$

Let $T \uparrow T_{\nu}$ and $\varphi \in C_{0}^{\infty}(\mathbf{R})$ sufficiently close to $u_{0}$ in $X^{s}$. Then, we have

$$
2 \tilde{C}_{\varphi} \delta_{0} \leq \tilde{C}_{\varphi} \delta_{0}+(1 / 2) \tilde{C}_{\varphi} \delta_{0}+C_{\varphi} T_{\nu}^{\beta}\left(1+2 \tilde{C}_{\varphi} \delta_{0}\right)^{3} .
$$

Hence, for any sequence $\left\{\nu_{n}\right\}$ such that $\nu_{n} \rightarrow 0$ as $n \rightarrow \infty, \liminf _{n \rightarrow \infty} T_{\nu_{n}}=0$ causes the contradiction. This is the proof of the first statement and (6.3) in Proposition 6.1. We next prove (6.4). Let $u_{\nu, \nu^{\prime}}=u_{\nu}-u_{\nu^{\prime}}$ and $v_{\nu, \nu^{\prime}}=v_{\nu}-v_{\nu^{\prime}}$. We see that

$$
\begin{aligned}
(6.20) u_{\nu, \nu^{\prime}}= & -G(t)\left(u_{\nu, \nu^{\prime}} \eta_{\nu} * \partial_{x} u_{\nu}\right)-G(t)\left(u_{\nu^{\prime}} \eta_{\nu^{\prime}} * \partial_{x} u_{\nu, \nu^{\prime}}\right) \\
& -G(t) u_{\nu^{\prime}}\left(\eta_{\nu}-\eta_{\nu^{\prime}}\right) * \partial_{x} u_{\nu} \\
(6.21) v_{\nu, \nu^{\prime}}= & V(t)\left(K_{\nu}-K_{\nu^{\prime}}\right) u_{0} \\
& -G(t) K_{\nu^{\prime}}\left(u_{\nu, \nu^{\prime}} \eta_{\nu} * \partial_{x} u_{\nu}\right)-G(t) K_{\nu^{\prime}}\left(u_{\nu^{\prime}}-\varphi\right) \eta_{\nu^{\prime}} * \partial_{x} u_{\nu, \nu^{\prime}} \\
& -G(t)\left(K_{\nu}-K_{\nu^{\prime}}\right)\left(\left(u_{\nu}-\varphi\right) \eta_{\nu} * \partial_{x} u_{\nu}\right) \\
& -G(t) K_{\nu^{\prime}}\left(\left(u_{\nu^{\prime}}-\varphi\right)\left(\eta_{\nu}-\eta_{\nu^{\prime}}\right) * \partial_{x} u_{\nu}\right) \\
& -G(t)\left(R_{\nu}\left(\varphi, u_{\nu}\right)-R_{\nu^{\prime}}\left(\varphi, u_{\nu^{\prime}}\right)\right)
\end{aligned}
$$


Applying Lemmas 6.1, 4.2 and the nonlinear estimates as in Lemma 5.1 to (6.20), we have

$$
\begin{gathered}
\left\|u_{\nu, \nu^{\prime}}\right\|_{L_{T}^{\infty}\left(H_{x}^{s_{1}^{\prime}, \alpha_{1}}\right)}+\left\|\left\langle D_{x}\right\rangle^{\mu^{\prime}}\langle x\rangle^{\alpha_{1}} u_{\nu, \nu^{\prime}}\right\|_{L_{x}^{2}\left(L_{T}^{\infty}\right)} \\
\leq C T^{\beta}\left(\left\|u_{\nu}\right\|_{Y_{T}^{\prime}}+\left\|u_{\nu^{\prime}}\right\| \|_{T}^{\prime}\right)\left\|u_{\nu, \nu^{\prime}}\right\|_{Y_{T}^{\prime}} \\
\quad+C T^{\beta}\left\|u_{\nu^{\prime}}\right\|_{Y_{T}^{\prime}}\left\|\left(\eta_{\nu}-\eta_{\nu^{\prime}}\right) * u_{\nu}\right\|_{Y_{T}^{\prime}} .
\end{gathered}
$$

In order to estimate $\left\|\left(\eta_{\nu}-\eta_{\nu^{\prime}}\right) * u_{\nu}\right\|_{Y_{T}^{\prime}}$ in (6.22), it suffices to show that

$$
\langle x\rangle^{\alpha}\left\langle D_{x}\right\rangle^{\sigma}\left(\eta_{\nu}-\eta_{\nu^{\prime}}\right) *\left\langle D_{x}\right\rangle^{-\tilde{\sigma}}\langle x\rangle^{-\alpha} \in \mathcal{B}\left(L_{T}^{r}\left(L_{x}^{p}\right)\right) \cap \mathcal{B}\left(L_{x}^{p}\left(L_{T}^{r}\right)\right),
$$

with the operator norms bounded by $C\left(\nu^{\beta}+\nu^{\prime \beta}\right)$, where $-1<\alpha<1,0<\sigma<$ $\tilde{\sigma}<2$ and $1 \leq p, r \leq \infty$. Note that $\eta_{\nu} *=\sqrt{2 \pi} \hat{\eta}\left(\nu i^{-1} \partial_{x}\right)$ and write

$$
\begin{aligned}
\langle x\rangle^{\alpha} & \left\langle D_{x}\right\rangle^{\sigma}\left(\eta_{\nu}-\eta_{\nu^{\prime}}\right) *\left\langle D_{x}\right\rangle^{-\tilde{\sigma}}\langle x\rangle^{-\alpha} \\
= & \sqrt{2 \pi}\langle x\rangle^{\alpha}\left(\hat{\eta}\left(\nu i^{-1} \partial_{x}\right)-\hat{\eta}\left(\nu^{\prime} i^{-1} \partial_{x}\right)\right)\left\langle D_{x}\right\rangle^{-(\tilde{\sigma}-\sigma)}\langle x\rangle^{-\alpha} \\
= & \sqrt{2 \pi}\left(\hat{\eta}\left(\nu i^{-1} \partial_{x}\right)-\hat{\eta}\left(\nu^{\prime} i^{-1} \partial_{x}\right)\right)\left\langle D_{x}\right\rangle^{-(\tilde{\sigma}-\sigma)} \\
& +\sqrt{2 \pi}\left[\langle x\rangle^{\alpha},\left(\hat{\eta}\left(\nu i^{-1} \partial_{x}\right)-\hat{\eta}\left(\nu^{\prime} i^{-1} \partial_{x}\right)\right)\left\langle D_{x}\right\rangle^{-(\tilde{\sigma}-\sigma)}\right]\langle x\rangle^{-\alpha} \\
\equiv & P_{1, \nu, \nu^{\prime}}\left(x, i^{-1} \partial_{x}\right)+P_{2, \mu, \mu^{\prime}}\left(x, i^{-1} \partial_{x}\right)\langle x\rangle^{-\alpha}
\end{aligned}
$$

Let us only consider the case $\alpha>0$ since, in this case, the multiplication of $\langle x\rangle^{-\alpha}$ is bounded on $L_{T}^{r}\left(L_{x}^{p}\right)\left(L_{x}^{p}\left(L_{T}^{r}\right)\right)$ and the other case $\alpha \leq 0$ is also verified by taking the commutator of $\left(\hat{\eta}\left(\nu i^{-1} \partial_{x}\right)-\hat{\eta}\left(\nu^{\prime} i^{-1} \partial_{x}\right)\right)\left\langle D_{x}\right\rangle^{-(\tilde{\sigma}-\sigma)}$ and $\langle x\rangle^{-\alpha}$. It is easy to see that $P_{1, \nu, \nu^{\prime}}(x, \xi) \in S^{-(\tilde{\sigma}-\sigma-\beta)}$ with $0<\beta<\min \{1, \tilde{\sigma}-\sigma\}$ and

$$
\begin{aligned}
\left|P_{1, \nu, \nu^{\prime}}\right|_{N}^{(-(\tilde{\sigma}-\sigma-\beta))} & \leq C_{N}\left|\hat{\eta}(\nu \xi)-\hat{\eta}\left(\nu^{\prime} \xi\right)\right|_{N^{\prime}}^{(\beta)} \\
& \leq C_{N}\left(|\hat{\eta}(\nu \xi)-1|_{N^{\prime}}^{(\beta)}+\left|\hat{\eta}\left(\nu^{\prime} \xi\right)-1\right|^{(\beta)_{N^{\prime}}}\right) \\
& \leq C_{N}\left(\nu^{\beta}+\nu^{\prime \beta}\right)
\end{aligned}
$$

where, to obtain the last inequality in the above estimate, we used $|\hat{\eta}(\nu \xi)-1| \leq$ $C \nu^{\beta}\langle\xi\rangle^{\beta}$ and $\left|\nu^{j}\left(\partial_{\xi}^{j} \hat{\eta}\right)(\nu \xi)\right| \leq C_{j} \nu^{\beta}\langle\xi\rangle^{-j+\beta}$. As for $P_{2, \nu, \nu^{\prime}}\left(x, i^{-1} \partial_{x}\right)$, we first note that

$$
\begin{aligned}
P_{2, \nu, \nu^{\prime}}(x, \xi) & =\frac{i}{\sqrt{2 \pi}} \iint e^{-i(x-y)(\xi-\zeta)} \partial_{\zeta}(\hat{\eta}(\nu \zeta) \\
& \left.\quad-\hat{\eta}\left(\nu^{\prime} \zeta\right)\right)\left.\langle\zeta\rangle^{-(\tilde{\sigma}-\sigma)} \int_{0}^{1} \partial_{w}\langle w\rangle^{\alpha}\right|_{w=\theta y+(1-\theta) x} d \theta d y d \zeta \\
\in & S^{-(1+\tilde{\sigma}-\sigma)}
\end{aligned}
$$


In particular, by regarding $P_{2}(x, \xi) \in S^{-(1+\tilde{\sigma}-\sigma-\beta)}$,

$$
\begin{aligned}
\left|P_{2, \nu, \nu^{\prime}}\right|_{N}^{(-(1+\tilde{\sigma}-\sigma-\beta))} & \leq C_{N}\left|\partial_{\xi}\left(\hat{\eta}(\nu \xi)-\hat{\eta}\left(\nu^{\prime} \xi\right)\right)\langle\xi\rangle^{-(\tilde{\sigma}-\sigma)}\right|_{N^{\prime}}^{(-(1+\tilde{\sigma}-\sigma-\beta))} \\
& \leq C_{N}\left|\hat{\eta}(\nu \xi)-\hat{\eta}\left(\nu^{\prime} \xi\right)\right|_{N^{\prime \prime}}^{(\beta)} \\
& \leq C_{N}\left(|\hat{\eta}(\nu \xi)-1|_{N^{\prime \prime}}^{(\beta)}+\left|\hat{\eta}\left(\nu^{\prime} \xi\right)-1\right|_{N^{\prime \prime}}^{(\beta)}\right) \\
& \leq C_{N}\left(\nu^{\beta}+\nu^{\prime \beta}\right) .
\end{aligned}
$$

Therefore, observing the integral kernels of $P_{j, \nu, \nu^{\prime}}\left(x, i^{-1} \partial_{x}\right)(j=1,2)$ and noting that these kernels are estimated in terms of some semi-norms of $P_{j, \nu, \nu^{\prime}}(x, \xi)$, we see that

$$
\begin{aligned}
& \left\|\langle x\rangle^{\alpha}\left\langle D_{x}\right\rangle^{\sigma}\left(\eta_{\nu}-\eta_{\nu^{\prime}}\right) *\left\langle D_{x}\right\rangle^{-\tilde{\sigma}}\langle x\rangle^{-\alpha}\right\|_{\mathcal{B}\left(L_{T}^{r}\left(L_{x}^{p}\right)\right) \cap \mathcal{B}\left(L_{x}^{p}\left(L_{T}^{r}\right)\right)} \\
& \quad \leq C\left(\nu^{\beta}+\nu^{\prime \beta}\right) .
\end{aligned}
$$

This implies that $\left\|\left(\eta_{\nu}-\eta_{\nu^{\prime}}\right) * u_{\nu}\right\|_{Y_{T}^{\prime}} \leq C\left(\nu^{\beta}+\nu^{\prime \beta}\right)\left\|u_{\nu}\right\|_{Y_{T}}$. Hence, by (6.22), we have

$$
\begin{aligned}
\left\|u_{\nu, \nu^{\prime}}\right\|_{L^{\infty}\left(H_{x}^{s_{1}^{\prime}, \alpha_{1}}\right)}+\left\|\left\langle D_{x}\right\rangle^{\mu^{\prime}}\langle x\rangle^{\alpha_{1}} u_{\nu, \nu^{\prime}}\right\|_{L_{x}^{2}\left(L_{T}^{\infty}\right)} \\
\leq C T^{\beta}\left(\left\|u_{\nu}\right\|_{Y_{T}}+\left\|u_{\nu^{\prime}}\right\| Y_{T}\right)\left\|u_{\nu, \nu^{\prime}}\right\|_{Y_{T}} \\
\quad+C\left(\nu^{\beta}+\nu^{\beta}\right)\left\|u_{\nu}\right\|_{Y_{T}}\left\|u_{\nu^{\prime}}\right\|_{Y_{T}} .
\end{aligned}
$$

Applying Lemma 3.1 and the nonlinear estimates as in Lemmas 5.1-5.2 with $s$ replaced by $s^{\prime}$ to (6.21) and making use of the estimates similar to (6.23) with $\left(\eta_{\nu}-\eta_{\nu^{\prime}}\right) *$ replaced by $K_{\nu}-K_{\nu^{\prime}}$, we see that

$$
\begin{aligned}
\| v_{\nu, \nu^{\prime}} & \left\|_{L_{T}^{\infty}\left(H_{x}^{s^{\prime}, 0}\right)}+\right\|\langle x\rangle^{-\rho}\left\langle D_{x}\right\rangle^{s^{\prime}+1 / 2} v_{\nu, \nu^{\prime}} \|_{L_{x}^{1 / \varepsilon}\left(L_{T}^{2}\right)} \\
\leq & C L\left(\varphi, u_{\nu}, u_{\nu^{\prime}}\right)\left\|u_{\nu, \nu^{\prime}}\right\| \|_{T}^{\prime} \\
& +C_{\varphi} T^{\beta}\left(1+\left\|u_{\nu}\right\|_{Y_{T}}+\left\|u_{\nu^{\prime}}\right\| \|_{Y_{T}}\right)\left\|u_{\nu, \nu^{\prime}}\right\|_{Y_{T}^{\prime}} \\
& +C_{\varphi}\left(\nu^{\beta}+\nu^{\prime \beta}\right)\left(1+\left\|u_{\nu}\right\|_{Y_{T}}+\left\|u_{\nu^{\prime}}\right\|_{Y_{T}}\right)^{2},
\end{aligned}
$$

where

$$
\begin{aligned}
L\left(\varphi, u_{\nu}, u_{\nu^{\prime}}\right)=\| & u_{\nu}\left\|_{\text {smooth }}+\right\| u_{\nu^{\prime}}-\varphi \|_{\text {maxim }} \\
& \quad+\left\|u_{\nu}\right\|_{\text {maxim }}^{1-\theta^{\prime}}\left\|u_{\nu}\right\|_{\text {smooth }}^{\theta^{\prime}}+\left\|u_{\nu^{\prime}}-\varphi\right\|_{\text {maxim }}^{\theta^{\prime}}\left\|u_{\nu^{\prime}}\right\|_{\text {smooth }}^{1-\theta^{\prime}}
\end{aligned}
$$

with $\theta^{\prime} \in(0,1)$ determined by $s^{\prime}-1 / 2=\theta^{\prime}\left(\mu^{\prime} / 2\right)+\left(1-\theta^{\prime}\right)\left(s^{\prime}+1 / 2-\mu^{\prime} / 2\right)$ for small $\mu^{\prime}>0$. Then, it follows from Lemmas $6.2-6.3$ that, by taking $\varphi$ close to $u_{0}$ and $T>0$ small in $(6.24)-(6.25)$,

$$
\left\|u_{\nu, \nu^{\prime}}\right\|_{Y_{T}^{\prime}} \leq \frac{1}{2}\left\|u_{\nu, \nu^{\prime}}\right\|_{Y_{T}^{\prime}}+C_{\varphi}\left(\nu^{\beta}+\nu^{\prime \beta}\right) .
$$


This completes the proof of (6.4).

We are now in the position to prove our main result.

Proof of Theorem 1.1. By Proposition 6.1 (6.3), there exist a function $u \in Y_{T}$ and a sequence $\left\{u_{\nu_{n}}\right\}$ such that

$$
\begin{aligned}
& \lim _{n \rightarrow \infty} u_{\nu_{n}}=u \quad \text { weakly-* in } L_{T}^{\infty}\left(H_{x}^{s, 0}\right) \text { and } L_{T}^{\infty}\left(H_{x}^{s_{1}, \alpha_{1}}\right), \\
& \lim _{n \rightarrow \infty}\langle x\rangle^{-\rho}\left\langle D_{x}\right\rangle^{s+1 / 2} u_{\nu_{n}}=\langle x\rangle^{-\rho}\left\langle D_{x}\right\rangle^{s+1 / 2} u \quad \text { weakly in } L_{x}^{1 / \varepsilon}\left(L_{T}^{2}\right), \\
& \lim _{n \rightarrow \infty}\left\langle D_{x}\right\rangle^{\mu}\langle x\rangle^{\alpha_{1}} u_{\nu_{n}}=\left\langle D_{x}\right\rangle^{\mu}\langle x\rangle^{\alpha_{1}} u \quad \text { weakly-* in } L_{x}^{2}\left(L_{T}^{\infty}\right) .
\end{aligned}
$$

Note that, in the above convergence, we identify $L_{T}^{\infty}\left(H_{x}^{s, 0}\right)\left(\operatorname{resp} . L_{T}^{\infty}\left(H_{x}^{s_{1}, \alpha_{1}}\right)\right.$, $\left.L_{x}^{2}\left(L_{T}^{\infty}\right)\right)$ with the dual of $L_{T}^{1}\left(H_{x}^{-s, 0}\right)$ (resp. $\left.L_{T}^{1}\left(H_{x}^{-s_{1},-\alpha_{1}}\right), L_{x}^{2}\left(L_{T}^{1}\right)\right)$. Furthermore, (6.4) implies that $\lim _{n \rightarrow \infty} u_{\nu_{n}}=u$ strongly in $Y_{T}^{\prime}$. Thus, the nonlinearity $u_{\nu_{n}} \eta_{\nu_{n}} * \partial_{x} u_{\nu_{n}}$ tends to $u \partial_{x} u$, for instance, in $L_{T}^{\infty}\left(L_{x}^{2}\right)$ and $u$ satisfies

$$
\partial_{t} u+\mathcal{H}_{x} \partial_{x}^{2} u+u \partial_{x} u=0 \quad \text { in } L_{T}^{\infty}\left(H_{x}^{s-2,0}\right) .
$$

Following the transformation as in section 2, we can write

$$
\begin{aligned}
& u=V(t) u_{0}-G(t) u \partial_{x} u, \\
& v=V(t) K_{0} u_{0}-G(t) K_{0}\left((u-\widetilde{\varphi}) \partial_{x} u\right)-G R_{0}(\widetilde{\varphi}, u),
\end{aligned}
$$

where $\widetilde{\varphi}$ is arbitrary $C_{0}^{\infty}$-function, $v=K_{0} u, K_{0}$ is the pseudo-differential operator of the symbol like

$$
K_{0}(x, \xi)=\exp \left(\sqrt{\frac{\pi}{2}} \frac{i \xi}{|\xi|}(1-\psi(\xi)) \int_{-\infty}^{x} \widetilde{\varphi}(y) d y\right),
$$

and $R_{0}(\widetilde{\varphi}, u)=K_{0} \widetilde{\varphi} \partial_{x} u+\left[K_{0}, \mathcal{H}_{x} \partial_{x}^{2}\right] u$.

Continuity in time. We next prove $u \in C\left([0, T] ; H_{x}^{s, 0} \cap H_{x}^{s_{1}, \alpha_{1}}\right)$. To this end, we use (6.27). Since $V(t) u_{0} \in C\left([0, T] ; H_{x}^{s, 0}\right)$, it sufficies to show that $G(t) u \partial_{x} u \in C\left([0, T] ; H_{x}^{s, 0}\right)$. Write

$$
\begin{aligned}
& \int_{0}^{t+h} V(t+h-\tau) u \partial_{x} u d \tau-\int_{0}^{t} V(t-\tau) u \partial_{x} u d \tau \\
& \quad=\int_{t}^{t+h} V(t+h-\tau) u \partial_{x} u d \tau+(V(h)-I) \int_{0}^{t} V(t-\tau) u \partial_{x} u d \tau \\
& \quad \equiv I_{1}(t, h)+I_{2}(t, h) .
\end{aligned}
$$

By Lemma 3.1, we have

$$
\left\|D_{x}^{s} I_{1}(t, h)\right\|_{L_{x}^{2}} \leq C\left\|D_{x}^{s-1 / 2}\left(u \partial_{x} u\right)\right\|_{L_{x}^{1}\left(L^{2}[t, t+h]\right)} .
$$


Therefore, Lebesgue's convergence theorem yields $\lim _{h \rightarrow 0} I_{1}(t, h)=0$. Since Lemma 3.1 also yields $\int_{0}^{t} V(t-\tau) u \partial_{x} u d \tau \in H_{x}^{s, 0}$, the strong continuity of $V(t)$ in $L_{x}^{2}$ gives $\lim _{h \rightarrow 0} I_{2}(t, h)=0$. Thus, $u \in C\left([0, T] ; H_{x}^{s, 0}\right)$. The continuity of $u$ in $H_{x}^{s_{1}, \alpha_{1}}$ follows by referring to Lemma 4.1.

Uniqueness in $Y_{T}$. Let $u_{1}, u_{2} \in Y_{T}$ be the solutions to (6.27) with the same initial data $u_{0}$. Note that $u_{j}(j=1,2)$ satisfies

$$
\begin{aligned}
& u_{j}=V(t) u_{0}-G(t) u_{j} \partial_{x} u_{j}, \\
& v_{j}=V(t) K_{0} u_{0}-G(t) K_{0}\left(\left(u_{j}-\widetilde{\varphi}\right) \partial_{x} u_{j}\right)-G(t) R_{0}\left(\widetilde{\varphi}, u_{j}\right) .
\end{aligned}
$$

Let $Y_{[0, h]}=\left\{u(t, x) ;\|u\|_{Y_{[0, h]}}<\infty\right\}$, where

$$
\begin{aligned}
\|u\|_{Y_{[0, h]}}= & \|u\|_{L^{\infty}\left([0, h] ; X^{s}\right)}+\left\|\langle x\rangle^{-\rho}\left\langle D_{x}\right\rangle^{s+1 / 2} u\right\|_{L_{x}^{1 / \varepsilon}\left(L^{2}[0, h]\right)} \\
& +\left\|\left\langle D_{x}\right\rangle^{\mu}\langle x\rangle^{\alpha_{1}} u\right\|_{L_{x}^{2}\left(L^{\infty}[0, h]\right)} .
\end{aligned}
$$

Applying the analogy to derive Proposition 6.1 (6.3) and taking $\widetilde{\varphi} \in C_{0}^{\infty}(\mathbf{R})$ close to $u_{0}$ and $h>0$ sufficiently small, we have $\left\|u_{1}-u_{2}\right\|_{Y_{[0, h]}} \leq 0$ i.e., $u_{1}(t)=u_{2}(t)$ on $0 \leq t \leq h$. By similar argument, we have $u_{1}(t)=u_{2}(t)$ on $0 \leq t \leq h+h^{\prime}$ for some $h^{\prime}>0$. Thus, the solution is unique in $Y_{T}$.

Stability. Let $u^{\prime}$ be the solution to (6.26) with $u_{0}^{\prime} \in X^{s}$ as initial data. Then, applying the similar argument for Proposition 6.1 to (6.27) and (6.28) with the common $\widetilde{\varphi} \in C_{0}^{\infty}(\mathbf{R})$ close to $u_{0}$ in $X^{s}$, we see that

$$
\left\|u^{\prime}-u\right\|_{Y_{T^{\prime}}} \leq C\left\|u_{0}^{\prime}-u_{0}\right\|_{X^{s}}+M\left(T^{\prime}, \widetilde{\varphi}, u^{\prime}, u\right)\left\|u^{\prime}-u\right\|_{Y_{T^{\prime}}},
$$

where

$$
\begin{aligned}
M\left(T^{\prime}, \widetilde{\varphi}, u^{\prime}, u\right)= & \left\|u^{\prime}\right\|_{\text {smooth }}+\|u\|_{\text {smooth }}+\left\|u^{\prime}\right\|_{\text {smooth }}^{\theta}\left\|u^{\prime}\right\|_{\text {maxim }}^{1-\theta} \\
& +\|u-\widetilde{\varphi}\|_{\text {maxim }}+C_{\widetilde{\varphi}} T^{\prime \beta}\left(1+\left\|u^{\prime}\right\|_{Y_{T^{\prime}}}+\|u\|_{Y_{T^{\prime}}}\right)
\end{aligned}
$$

for some $\theta \in(0,1)$. By Lemma $6.3, M\left(T^{\prime}, \widetilde{\varphi}, u^{\prime}, u\right)$ is estimated as

$$
\begin{aligned}
M\left(T^{\prime}, \widetilde{\varphi}, u^{\prime}, u\right) \leq & C\left(\left\|u_{0}^{\prime}-\widetilde{\varphi}\right\|_{X^{s}}+\left\|u_{0}-\widetilde{\varphi}\right\|_{X^{s}}\right) \\
& +C_{\widetilde{\varphi} T^{\prime \beta^{\prime}}}\left(1+\left\|u^{\prime}\right\|_{Y_{T^{\prime}}}+\|u\|_{Y_{T^{\prime}}}\right)^{N},
\end{aligned}
$$

where $\beta^{\prime}, N>0$ and $C_{\widetilde{\varphi}}>0$ may diverge as $\widetilde{\varphi} \rightarrow u_{0}$ in $X^{s}$. If $u_{0}^{\prime}$ is sufficiently close to $u_{0}$, then, by taking $\widetilde{\varphi} \in C_{0}^{\infty}$ close to $u_{0}$ and $T^{\prime}>0$ small enough, we can make $M\left(T^{\prime}, \widetilde{\varphi}, u^{\prime}, u\right)$ small. This implies Theorem 1.1(ii). 


\section{References}

[1] Abdelouhab, L., Bona, J. L., Felland, M. and Saut, J.-C., Nonlocal models for nonlinear dispersive waves, Phys. D, 40 (1989), 360-392.

[2] Bekiranov, D., Ogawa, T. and Ponce, G., On the well-posedness of Benney's interaction equation of short and long waves, Adv. Differential Equations, 1 (1996), 919-937.

[3] Benjamin, T. B., Internal waves of permanent form in fluids of great depth, J. Fluid. Mech., 29 (1967), 559-592.

[4] Bergh, J. and Löfström, J., Interpolation spaces. An introduction, Springer-Verlag, Berlin-New York, 223 (1976).

[5] Biagioni, H. A. and Linares, F., Ill-posedness for the derivative Schrödinger and generalized Benjamin-Ono equations, Trans. Amer. Math. Soc., 353 (2001), 3649-3659.

[6] Ginibre, J. and Velo, G., Scattering theory in the energy space for a class of nonlinear schrödinger equations, J. Math. Pures Appl., 64 (1985), 363-401.

[7] Smoothing properties andexistence of solutions for the generalized BenjaminOno equation, J. Differential Equations, 93 (1991), 150-212.

[8] Iorio, R. Jr., On the Cauchy problem for the Benjamin-Ono equation, Comm. Partial Differential Equations, 11 (1986), 1031-1081.

[9] Kato, K., On the existence of solutions to the Benjamin-Ono equation, Preprint 2004.

[10] Kenig, C. E. and Koenig, K., On the local well-posedness of the Benjamin-Ono and modified Benjamin-Ono equations, Math. Res. Lett., 10 (2003), 879-895.

[11] Kenig, C. E., Ponce, G. and Vega, L., Oscillatory integrals and regularity of dispersive equations, Indiana Univ. Math. J., 40 (1991), 33-69.

[12] Well-posedness and scattering results for the generalized Korteweg-de Vries equation via the contraction principle, Comm. Pure Appl. Math., 46 (1993), 527-620.

[13] , On the generalized Benjamin-Ono equation, Trans. Amer. Math. Soc., 342 (1994), 155-172.

[14] Koch, H. and Tzvetkov, N., On the local well-posedness of the Benjamin-Ono equation, Int. Math. Res. Not. (2003), 1449-1464.

[15] - Nonlinear wave interactions for the Benjamin-Ono equation, Preprint 2003.

[16] Kumano-go, H., Pseudo-differential oparators, The MIT Press, 1981.

[17] Molinet, L., Saut, J. C. and Tzvetkov, N., Ill-posedness issues for the Benjamin-Ono and related equations, SIAM J. Math. Anal., 33 (2001), 982-988.

[18] Ono, H., Algebraic solitary waves in stratified fluids, J. Phys. Soc. Japan, 39 (1975), 1082-1091.

[19] Ponce, G., On the global well-posedness of the Benjamin-Ono equation, Differential Integral Equations, 4 (1991), 527-542.

[20] Stein, E. M., Harmonic analysis: real-variable methods, orthogonality and oscillatory integrals, Princeton University Press, Princeton, NJ, 1993.

[21] Saut, J-C., Sur quelques généralisations de l'équation de Korteweg-de Vries. (French) $J$. Math. Pures Appl., 58 (1979), 21-61.

[22] Tao, T., Global well-posedness of Benjamin-Ono equation in $H^{1}(\mathbf{R}), \quad J$. Hyperbolic Differ. Equ., 1 (2004), 27-49.

[23] Tom, M. M., Smoothing properties of some weak solutions of the Benjamin-Ono equation, Diff. Int. Eq., 3 (1990), 683-694.

[24] Vega, L., Doctral thesis, Univ. Autonoma de Madrid, Spain, 1987.

[25] Yajima, K., Existence of solutions for Schrödinger evolution equations, Comm. Math. Phys., 110 (1987), 415-426. 